\title{
A Hybrid Commodity and Interest Rate Market Model
}

\author{
K.F. Pilz and E. Schlögl* \\ University of Technology \\ Sydney \\ Australia
}

September 15, 2011

\begin{abstract}
A joint model of commodity price and interest rate risk is constructed analogously to the multi-currency LIBOR Market Model (LMM). Going beyond a simple "re-interpretation" of the multi-currency LMM, issues arising in the application of the model to actual commodity market data are specifically addressed. Firstly, liquid market prices are only available for options on commodity futures, rather than forwards, thus the difference between forward and futures prices must be explicitly taken into account in the calibration. Secondly, we construct a procedure to achieve a consistent fit of the model to market data for interest options, commodity options and historically estimated correlations between interest rates and commodity prices. We illustrate the model by an application to real market data and derive pricing formulas for commodity spread options.
\end{abstract}

Keywords: Commodity modeling, LIBOR Market Model, commodity futures, interest rate risk, spread options

\section{Introduction}

Traditionally, market risks in different asset classes such as FX, fixed income or commodities, have been modelled separately, though modelling approaches incorporating several sources of

\footnotetext{
${ }^{*}$ This research was supported as part of a project with SIRCA under the Australian Research Council's Linkage funding scheme (project number LP0562616). The authors would like to thank the Editors of Quantitative Finance and two anonymous referees for helpful comments. The usual disclaimer applies. Corresponding author: E. Schlögl, School of Finance and Economics, University of Technology, Sydney, PO Box 123, Broadway, NSW 2007, Australia. E-mail: Erik.Schlogl@uts.edu.au
} 
market risk are becoming increasingly popular. The aim of this paper is to contribute to the more integrated approach by applying the multi-currency LIBOR Market Model (LMM) as presented in Schlögl [19] jointly to interest rates and commodities. The domestic fixed income market will be interpreted in the usual way for the LMM, i.e. with a given bond market paying in a certain currency (say USD), whereas the foreign market will be associated with a commodity market (e.g. crude oil), with the physical commodity as its currency, and the "convenience yield" as its rate of interest. The contribution of this paper goes beyond a simple "re-interpretation" of the multicurrency LMM in that issues arising in the application of the model to actual commodity market data are specifically addressed. Firstly, liquid market prices are only available for options on commodity futures, rather than forwards, thus the difference between forward and futures prices must be explicitly taken into account in the calibration. Secondly, we construct a procedure to achieve a consistent fit of the model to market data for interest rate options, commodity options and historically estimated correlations between interest rates and commodity prices. This is achieved in a two-stage process, calibrating first to the interest rate and commodity markets separately, followed by an orthonormal transformation of the commodity volatility vectors to "rotate" the commodity volatilities relative to the interest rate volatilities in such a manner as to achieve the desired correlations between the two markets. Thirdly, we illustrate the use of the model on actual market data and demonstrate a way of efficiently calculating commodity spread options.

For the market data illustration, we chose WTI Crude Oil as the commodity and US dollars as the "domestic" currency. Thus the "exchange rate" is the WTI Crude Oil price, i.e. the WTI Crude Oil futures quotes - when converted to forward prices using an appropriate convexity correction - can be interpreted as forward exchange rates between the US dollar economy and an economy where value is measured in terms of units of WTI Crude Oil (and where convenience yields play the role of interest rates). In this example, the model is calibrated to USD at-themoney caplets and swaptions, as well as WTI Crude Oil futures and ATM options on futures. The model presented here builds on the prior literature on the LMM, starting from the seminal work of Miltersen, Sandmann and Sondermann [13], Brace, Gatarek and Musiela [3] and Jamshidian [9]. The model construction follows Musiela and Rutkowski [15] and the calibration method builds on the approach first proposed by Pedersen [16] for the basic LMM.

The earliest work combining commodity and interest rate risk (based on dynamics of the continuously compounded short rate, without reference to a full model calibrated to an initial term structure) goes back to Schwartz [20]. Subsequently, models for commodity price dynamics incorporating stochastic convenience yields have been constructed by a number of authors, for example Gibson and Schwartz [7], Cortazar and Schwartz [5], Schwartz [21], Miltersen and Schwartz [14], and Miltersen [12]. These models are typically constructed on the basis of a Heath, Jarrow and Morton [8] term structure model with generalised (possibly multi-factor) Ornstein/Uhlenbeck dynamics, i.e. continuously compounded convenience yields (and possibly interest rates rates) are normally distributed. While in theory these models allow some freedom to calibrate to market data, the published work does not contain much evidence as to whether 
an effective calibration to available commodity and interest rate options - even at the money - can be achieved. This type of fit to observed market prices, in particular to at-the-money options, is a strength of the LIBOR Market Models, which we exploit in the present paper.

The paper is organised as follows. The basic notation, the results of the single- and multicurrency LMM and their interpretation in the context of commodities are presented in Section 2. In Section 3 the calibration of the commodity part of the Commodity LMM to plain vanilla options is discussed. In all major commodity markets, only options with the same maturity as the underlying forward are traded liquidly. Hence, no volatility term structure for a particular forward commodity price can be extracted only from the options written on this forward. To overcome this problem, option prices for all maturities are used to calibrate simultaneously a volatility term structure, which is desired to be as time-homogeneous and smooth as possible. In keeping with the assumptions of the LMM, we thus focus on the volatility term structure only, i.e. with time dependence but no strike dependence. In Section 4 the relationship between futures and forwards in the model is presented, which permits calibration of the model to futures as well as forwards. The calibration of the interest rate part of the hybrid Commodity LMM will not be discussed in detail in this paper, because this problem has already been addressed by many authors and most methods should be compatible with our model. However, in Section 5 we discuss how both separately calibrated parts - the interest rate and the commodity part - of the model can be merged in order to have one underlying $d$-dimensional Brownian motion for the joint model and still match the market prices used for calibration of the particular parts. Section 6 illustrates the application of the Commodity LMM to real market data. Finally, Section 7 addresses the pricing of commodity spread options in the model.

\section{The Commodity LIBOR Market Model}

\subsection{The Interest Rate Market}

The construction of the LMM for the domestic interest rate market follows the presentation in Musiela and Rutkowski [15]. Assume a given probability space $\left(\Omega,\left\{\mathcal{F}_{t}\right\}_{t \in\left[0, T^{*}\right]}, \mathbb{P}\right)$, where the underlying filtration $\left\{\mathbb{F}_{t}\right\}_{t \in\left[0, T^{*}\right]}$ coincides with the $\mathcal{P}$-augmentation of the natural filtration of a $d$-dimensional standard Brownian motion $W$. Let $T^{*}$ be a fixed time horizon, then a family of bond prices is any family of strictly positive real-valued adapted processes $B(t, T)$ for $t \in[0, T]$, with $B(T, T)=1$ for every $T \in\left[0, T^{*}\right]$. The bond price $B(t, T)$ for $T \in\left[t, T^{*}\right]$ is the amount that has to be invested at time $t$ to receive one unit of the domestic currency at time $T$.

Corresponding to the assumptions (BP.1) and (BP.2) in Musiela and Rutkowski [15], we have that the bond price $B(t, T)$ for $t \leq T \leq T^{*}$ is a strictly positive special semi-martingale (see Musiela and Rutkowski [15], p. 263 for the definition) and that the forward process

$$
F_{B}\left(t, T, T^{*}\right):=\frac{B(t, T)}{B\left(t, T^{*}\right)}
$$


follows a martingale under the $T^{*}$-forward measure $\mathbb{P}$. Equivalently, the bond price process satisfies

$$
B(t, T)=\mathbb{E}_{\mathbb{P}}\left[\frac{B\left(t, T^{*}\right)}{B\left(T, T^{*}\right)} \mid \mathcal{F}_{t}\right]
$$

for all $t \in[0, T]$.

We present some conclusions from these assumptions, which will be of use later and can be found in Musiela and Rutkowski [15]. Firstly, there exists a $\mathbb{R}^{d}$-valued process $\gamma\left(t, T, T^{*}\right)$, such that the forward process has the representation

$$
d F_{B}\left(t, T, T^{*}\right)=F_{B}\left(t, T, T^{*}\right) \gamma\left(t, T, T^{*}\right) \cdot d W_{T^{*}}(t) .
$$

By Itô's formula and Girsanov's theorem it follows, that for any given $S, T \in\left[0, T^{*}\right]$ the dynamics of $F_{B}(t, S, T)$ for $t \in[0, S \wedge T]$ can be written as

$$
d F_{B}(t, S, T)=F_{B}(t, S, T) \gamma(t, S, T) \cdot d W_{T}(t),
$$

with

$$
\begin{aligned}
\gamma(t, S, T) & =\gamma\left(t, S, T^{*}\right)-\gamma\left(t, T, T^{*}\right) \\
d W_{T}(t) & =d W_{T^{*}}(t)-\gamma\left(t, T, T^{*}\right) d t .
\end{aligned}
$$

Hence, $F_{B}(t, S, T)$ is an exponential (local) martingale under the $\mathbb{P}_{T}$ measure, and since both, $\mathbb{P}$ and $\mathbb{P}_{T}$, are absolutely continuous, the Radon-Nikodým derivative is given by the Doléans exponential

$$
\frac{d \mathbb{P}_{T}}{d \mathbb{P}}=\mathcal{E}_{T}\left(\int_{0} \gamma\left(u, T, T^{*}\right) \cdot d W_{T^{*}}(u)\right), \quad \mathbb{P}_{T}-\text { a.s. }
$$

The measure $\mathbb{P}_{T}$ is usually denoted as $T$-forward measure, and $\mathbb{P}_{T^{*}}=\mathbb{P}$ additionally as terminal measure.

For practical purposes it is often convenient to use a discrete-tenor version of the LMM ${ }^{1}$. Therefore, we assume that the time horizon $T^{*}=N \delta$ is a multiple of a fixed period $\delta$. Then, the LIBOR forward rate $L(t, T)$ as seen at time $t$, for an investment of one currency unit at time $T$ with payoff $B(t, T) / B(t, T+\delta)$ at $T+\delta$, can be written as

$$
d L(t, T)=L(t, T) \lambda(t, T) \cdot d W_{T}(t),
$$

for $t \in[0, T]$. The volatility function of LIBOR relates to the volatility function of the forward process by

$$
\gamma(t, T, T+\delta)=\frac{\delta L(t, T)}{1+\delta L(t, T)} \lambda(t, T)
$$

To shorten notation, define $T_{i}=i \delta$ (for $\left.0 \leq i \leq N\right)$.

\footnotetext{
${ }^{1}$ Where an extension to continuous tenor is required, it is typically more practical to start with a discrete-tenor version of the LMM and extend it using "interpolation by daycount fractions" as in Schlögl [18] in order to avoid the infinite-dimensional state variables resulting from the continuous-tenor extensions proposed by Brace, Gatarek and Musiela [3] and Musiela and Rutkowski [15].
} 


\subsection{The Commodity Market}

The approach incorporating a commodity as followed in this section parallels the multi-currency LMM introduced by Schlögl [19]. The commodity market can naturally be considered as a "foreign interest rate market", where the currency is the physical commodity itself, e.g. as in our example in Section 6, the value of any asset is measured in units (barrels) of crude oil instead of dollars. The bond prices $C(t, T)$ quote (as seen at time $t$ ) the amount of the commodity that has to be invested at time $t$ to physically receive one unit of the commodity at time $T$. Corresponding LIBORs can be interpreted in the same way, i.e. the yield of $C(t, T)$ is the convenience yield (adjusted for storage costs, if applicable). Although these rates have a natural interpretation, they are usually not traded (liquidly) for most of the commodities. We will discuss below how this model still can be calibrated to the instruments commonly traded in the commodities market.

Some assumptions are required with regard to the commodity market. Firstly, all assumptions made in constructing the LMM on the domestic interest rate market in the previous section, are assumed to be true for the commodity market as well. This in particular includes the process $C(t, T)$ for $0 \leq t \leq T \leq T^{*}$ to be adapted to the filtration $\left\{\mathcal{F}_{t}\right\}_{t \in\left[0, T^{*}\right]}$. We will denote the corresponding $T_{i}$-forward measures, Brownian motions and volatility functions by $\tilde{\mathbb{P}}_{T_{i}}, \tilde{W}_{T_{i}}$ and $\tilde{\gamma}\left(t, T_{i}, T_{i+1}\right)$, respectively.

Secondly, we postulate the existence of a spot exchange rate process $X(t)$, which is a positive special semi-martingale under $\mathbb{P}_{T^{*}} . X(t)$ is the spot price of the commodity at time $t$.

A commodity bond $C(t, T)$ converted by the spot exchange rate is then a traded asset in the domestic market (denominated in domestic currency), and hence its forward value,

$$
X\left(t, T_{i}\right)=\frac{C\left(t, T_{i}\right) X(t)}{B\left(t, T_{i}\right)}, \quad(0 \leq i \leq N),
$$

is a martingale under $\mathbb{P}_{T_{i}}$ and is called $T_{i}$-forward exchange rate and in the present context this is the forward price of the commodity. If the dynamics of the forward exchanges rates are written in terms of

$$
d X\left(t, T_{i}\right)=X\left(t, T_{i}\right) \sigma_{X}\left(t, T_{i}\right) \cdot d W_{T_{i}}(t), \quad(0 \leq i \leq N),
$$

in which the volatility functions are not necessarily deterministic, then it is shown in Schlögl [19], that under no-arbitrage restrictions the volatility functions must satisfy the relation

$$
\sigma_{X}\left(t, T_{i-1}\right)=\tilde{\gamma}\left(t, T_{i-1}, T_{i}\right)-\gamma\left(t, T_{i-1}, T_{i}\right)+\sigma_{X}\left(t, T_{i}\right), \quad(1 \leq i \leq N)
$$

This leaves generally two ways to link the interest rate and commodity markets in order to have an arbitrage free multi-currency LMM. The first one would be to calibrate the single-currency LMM models separately, i.e. determining the volatility functions $\gamma\left(t, T_{i}, T_{i+1}\right)$ and $\tilde{\gamma}\left(t, T_{i}, T_{i+1}\right)$ (or $\lambda\left(t, T_{i}\right)$ and $\tilde{\lambda}\left(t, T_{i}\right)$ equivalently) for $i=1, \ldots, N-1$, and then calibrate the forward exchange 
rate volatility $\sigma_{X}\left(\cdot, T_{i}\right)$ for one arbitrary forward time $T_{i}$. Using (10) all other forward exchange rate volatilities $\sigma_{X}\left(\cdot, T_{j}\right)$ for $1 \leq j \neq i \leq N$ can be derived. This approach seems especially appropriate when linking two (real) interest rate markets (like USD and AUD), because LIBORs (or similar) and currency forwards are liquidly traded for all major currencies.

In order to link an interest rate market with a commodity market, the second approach seems to be more appropriate. The fixed income market volatilities $\gamma\left(t, T_{i}, T_{i+1}\right)$ (or equivalently $\lambda\left(t, T_{i}\right)$ ) are calibrated as for a single-currency LMM - in our example in Section 6, this will be the USD market calibrated to at-the-money caplets and swaptions. Then, for all forward times $T_{0}, \ldots, T_{N}$ volatility functions $\sigma_{X}\left(\cdot, T_{i}\right)$ are calibrated to the commodity options market (in Section 6, we specifically consider options on WTI Crude Oil futures), i.e. the forward commodity prices are assumed to follow lognormal dynamics under the appropriate probability measure, rather than the simple-compounded convenience yields. ${ }^{2}$ The volatility functions $\tilde{\gamma}\left(t, T_{i}, T_{i+1}\right)$ and $\tilde{\lambda}\left(t, T_{i}\right)$ can now be derived from relations (10) and (7), respectively.

Remark 2.1 If a deterministic volatility function $\sigma_{X}$ is chosen for the commodity forward process, it will be shown in Section 4 that the corresponding futures process is not log-normally distributed, apart from the futures price at maturity. Nevertheless, the difference between forward and futures prices can be expressed in terms of volatilities for the commodity forwards and forward interest rates.

\section{Calibration with Time Dependent Volatilities}

Since the Commodity LMM is based on commodity forwards, we have to calibrate to forward implied volatilities or plain vanilla option prices written on forwards. However, commodities futures rather than forwards are most liquidly traded (consider, for example, the WTI Crude Oil futures in the market data example in Section 6) and thus forward prices have to be deduced from the futures. As we are specifically concerned with integrating commodity and interest rate risk, it is not adequate to equate forward prices with futures prices, as is still common among practitioners. The following Section 4 describes how to approximate the difference between futures and forwards as well as the implied forward volatilities, in order to apply the calibration methods proposed in the present section.

We first make some notational conventions and modify slightly the notation in Section 2, adapted from the prior literature. Since in our setup the forward exchange rate $X(t, T)$ is a commodity forward, we will write $F(t, T)$ instead, where $t$ is termed process time (aka calendar time) and $T$ as forward time. Accordingly, the volatility of the forwards will be denoted by $\sigma(t, T)$, instead of $\sigma_{X}(t, T)$, and is the instantaneous volatility at time $t$ of the forward with forward time $T$. We assume to have forward processes $F\left(\cdot, T_{1}\right), \ldots, F\left(\cdot, T_{N}\right)$ with expiries $T_{1}, \ldots, T_{N}$ and we further think of $T_{0}$ as "now". Times to maturity for an arbitrary calendar time $t \geq 0$ are given

\footnotetext{
${ }^{2}$ Assuming the convenience yields to be lognormal also does not appear reasonable in light of the fact that, empirically, convenience yields can become negative.
} 
by $x_{i}=T_{i}-t$ for $i=0,1, \ldots, N$. Finally, market prices for call options on $F\left(\cdot, T_{i}\right)$ with payoff $\left[F\left(T_{i}, T_{i}\right)-K\right]^{+}$are assumed to be available and denoted by $C_{i}^{\text {mkt }}$ for $i=1, \ldots, N$.

For calibration we further need to determine vectors of calendar times $t_{c}=\left(0, t_{1}, \ldots, t_{n_{c}}\right)$ and times to maturity $x_{f}=\left(x_{0}, x_{1}, \ldots, x_{n_{f}}\right)$, which define a grid for $V=\left(v_{i, j}\right)_{1 \leq i \leq n_{c}, 1 \leq j \leq n_{f}}$, the matrix of piecewise constant instantaneous volatilities. The entry $v_{i, j}$ represents the volatility corresponding to forward $F(t, T)$ with $t_{i-1} \leq t<t_{i}$ and $x_{j-1} \leq T-t<x_{j}$. The number of forward times $n_{f}$ in the volatility matrix need not to coincide with the number of traded forwards $N$, and especially in regions of large forward times a rougher spacing can be chosen for $x_{f}$, since volatilities tend to flatten out with increasing forward time. Let $\nu(t, T)$ denote the (annualized) implied volatility as seen at time $t$ for forward time $T$, then the relation between time-dependent instantaneous and implied volatilities is given by

$$
\frac{1}{T-t} \int_{t}^{T} \sigma^{2}(s, T) d s=\nu^{2}(t, T)
$$

Identity (11) is utilized to compute a (model) option price $C_{i}^{\bmod }$ (for $i=1, \ldots, N$ ) from the volatility matrix $V$ with Black's formula [1]. Note, that the assumption of deterministic volatilities for commodity forwards made in the previous section permits us to apply Black's formula in a consistent manner. In Appendix A we show how to compute the integral when volatilities are piecewise constant. Now, the first calibration criterion measures the quality of fit by considering the squared differences between market and model call prices. In order to weight the various fit criteria we assign different weights $\eta$ to each of them,

$$
q=\eta_{q} \sum_{j=1}^{N}\left(C_{j}^{\mathrm{mkt}}-C_{j}^{\mathrm{mod}}\right)^{2} .
$$

As already mentioned in the introduction, in commodity markets typically only options with maturities coinciding with the maturity of the underlying forward exist. In order to construct a full volatility term structure for a particular forward, it is necessary to use information gained from other options on forwards expiring before the particular one. Following Pedersen [16], to this end several heuristic concepts can be employed, which more or less rely on market practice and experience. We will refer to these as smoothness criteria. The first one is time-homogeneity, which assumes that the (unknown) volatility at future calendar time $t=T_{2}-T_{1}$ of a forward $F\left(\cdot, T_{2}\right)$ having then time to maturity $T_{1}$ and time to maturity $T_{2}$ now, will be "similar" to the (known) volatility the forward $F\left(\cdot, T_{1}\right)$ with time to maturity $T_{1}$ has now. In other words, under strict time-homogeneity $\sigma(t, T)$ can be expressed as a function of time to maturity $T-t$ only. This is usually too restrictive to be able to fit the model to the market in general, so instead of enforcing strict time-homogeneity, we penalise departures from time-homogeneity in the first smoothness criterion. In terms of the volatility matrix $V$, this means that volatilities with different calendar times but the same time to maturity should not differ too much, so the 
penalty function is

$$
s_{1}=\eta_{1} \sum_{j=1}^{n_{f}} \sum_{i=1}^{n_{c}-1}\left(v_{i+1, j}-v_{i, j}\right)^{2} .
$$

Another typical characteristic is the so-called Samuelson effect, which states that forward volatilities tend to decrease with increasing time to maturity. In order to be consistent with this behavior, the volatility matrix has to be monotonically decreasing in forward time, and violations of this property are penalised by

$$
s_{2}=\eta_{2} \sum_{i=1}^{n_{c}} \sum_{j=1}^{n_{f}-1}\left(\max \left\{v_{i, j+1}-v_{i, j}, 0\right\}\right)^{2} .
$$

Finally, we enable a criterion which imposes the volatility term structure to be smooth in time to maturity for each fixed calendar time. Assigning a large weight to this criterion would force the volatility to be flat in the forward time direction, which is usually not desirable, however, with a small weight this criterion contributes to a smoother volatility surface,

$$
s_{3}=\eta_{3} \sum_{i=1}^{n_{c}} \sum_{j=1}^{n_{f}-1}\left(v_{i, j+1}-v_{i, j}\right)^{2} .
$$

For minimising of the objective value $q+s_{1}+s_{2}+s_{3}$ a least-squares optimisation method is used; one that allows for the non-linear dependencies between model parameters and objective function values.

Remark 3.1 The quality-of-fit criterion $q$ and the smoothness criteria $s_{1}, s_{2}, s_{3}$ are constructed such that absolute squared differences between model and market option prices and between neighboring volatilities, respectively, are minimised. Alternatively, relative deviations could be used for minimisation, but we did not experience substantial differences in the quality of fit or the smoothness of volatilities when doing so.

Remark 3.2 Regarding the relationship between calendar times and forward times of the volatility matrix and the times to maturity of the available options on forwards, two important points should be noted. Firstly, to be able to price options on all of the forwards, the maturity of the longest available forward has to be smaller or equal to the latest calendar time and the longest time to maturity, $T_{N} \leq \min \left\{t_{n_{c}}, x_{n_{f}}\right\}$. Here, we assume that all options mature at the same time as their underlying forwards. Secondly, in context of the integral in (11), volatilities $\sigma(t, T)$ at any calendar time $t$ with forward time $T>T_{N}$, or equivalently $t+x>T_{N}$, have no impact on the price of any plain vanilla option used for calibration. In terms of the piecewise constant volatility matrix this means that volatilities $v_{i, j}$ with $t_{i-1}+x_{j-1}>T_{N}$ have no contribution to the quality of fit criterion and are therefore determined only by smoothness criteria. 
We want to conclude this section by briefly describing the concept of volatility factor decomposition and factor reduction, which can either be included in the calibration process, or applied to the final volatility matrix after calibration. As it will be demonstrated in the following Sections 4 and 5, the volatility factor decomposition has to be included in the calibration process when calibrating to futures and options on futures. The method has to be applied separately for every calendar time, which is therefore fixed to arbitrary $t_{i}$ in the following, and let $v_{i}$ denote the $i$ th row (corresponding to calendar time $t_{i}$ ) of $V$, written as column vector. Together with the exogenously given correlation matrix $C$, which is assumed to be constant over calendar time, the covariance matrix is calculated by

$$
\Sigma=\left(v_{i} v_{i}^{\top}\right) \odot C
$$

where ${ }^{\top}$ means transposition and $\odot$ multiplication by components (Hadamard product). $\Sigma$ is symmetric and positive definite, hence can be decomposed into $\Sigma=R D^{1 / 2}\left(R D^{1 / 2}\right)^{\top}$. The columns of $R=\left(r_{j, k}\right)_{1 \leq j, k \leq n_{f}}$ consists of orthonormal eigenvectors and the diagonal matrix $D=\left(\xi_{j, k}\right)_{1 \leq j, k \leq n_{f}}$ of the eigenvalues of $\Sigma$. This representation allows us to reduce the number of stochastic factors, because usually the first $d$ eigenvalues (when ordered decreasingly in $D$ and the columns of $R$ accordingly) account for more than around $95 \%$ of the overall variance, with $d$ substantially smaller than $n_{f}$. Hence, $R$ and $D$ can be reduced to matrices $R \in \mathbb{R}^{n_{f} \times d}$ and $D \in \mathbb{R}^{d \times d}$ by retaining only the first $d$ columns in $R$ and the upper $d \times d$ sub-matrix in $D$, respectively. Instead of $v_{i}$ we now use the a matrix $U=\left(u_{j, k}\right)_{1 \leq j \leq n_{f}, 1 \leq k \leq d}$ (the calendar time index $i$ has been skipped for notational convenience) with entries $u_{j, k}=r_{j, k} \sqrt{\xi_{k}}$, which relates to $v_{i}$ by

$$
v_{i, j}^{2}=\sum_{k=1}^{d} u_{j, k}^{2}=\sum_{k=1}^{d} r_{j, k}^{2} \xi_{k} \quad\left(j=1, \ldots, n_{f}\right)
$$

The computation of the variance in (11), as described in Appendix A, allows also for representations of the volatilities in decomposed form.

Remark 3.3 The correlation matrix $C$ in (16) is the correlation matrix of the forward returns, not of the futures returns. ${ }^{3}$ However, for the convexity adjustment suggested in Section 4 both correlation matrices coincide.

Remark 3.4 When used as in (16), the calculation of a correlation matrix from a historical time series should be consistent with the forward time concept of the volatility matrix. That means, if the volatility matrix is given for calendar times $t$ and absolute forward times $T$, the correlations should be calculated from instruments with the same absolute maturity times. Correspondingly, if the volatility matrix is given for calendar times $t$ and times to maturity $x$, the correlations should be calculated from instruments with the same times to maturity. Typically, futures are

\footnotetext{
${ }^{3}$ Strictly speaking, it is the matrix of quadratic covariation of the forward processes.
} 
quoted in the market for absolute maturity times and forwards (if they are quoted at all) have constant times to maturities.

Although in practice both methods typically do not exhibit substantial differences, an interpolation could be performed before estimating the correlations in order to switch from absolute maturity times to times to maturity or vice versa. For a discussion of different correlation concepts and their effect on calibration and pricing in the context of the LMM, see [4].

\section{Futures/Forward Relation and Convexity Correction}

The calibration method in Section 3 is applicable only when forwards and options on forwards are available. This section presents the approximate conversion of futures prices to forward prices for all relevant data for calibration, in order to apply the methods of the previous section when only futures and options on futures are available (such as in the case of WTI Crude Oil as considered in the market data example in Section 6).

We introduce the notation $G(t, T)$ for a futures price at time $t$ with maturity $T$, and, as before, $F(t, T)$ will be the corresponding forward price. From no-arbitrage theory we know $F(T, T)=$ $G(T, T)$ and that prices of plain vanilla options on forwards and futures must coincide, whenever the maturities of option, forward and futures are the same. This allows us to use the call prices of options on futures for calibration of forwards, and we only have to assure that the (virtual) forwards have the same maturities as the futures. Due to equation (9) the forward $F\left(\cdot, T_{i}\right)$ is an exponential martingale under the $T_{i}$-forward measure, and since it has deterministic volatility, it is log-normally distributed under $\mathbb{P}_{T_{i}}$. A change of measure from the $T_{i}$-forward to the spot risk-neutral measure relates the Brownian motions by

$$
d W_{T_{i}}(t)=\eta\left(t, T_{i}\right) d t+d W_{\mathbb{Q}}(t) \quad(1 \leq i \leq N),
$$

and $\eta\left(\cdot, T_{i}\right)$ relates to the volatility of the process $F_{B}\left(t, T_{i}, T_{i}+\delta\right)$ by

$$
\eta\left(t, T_{i+1}\right)-\eta\left(t, T_{i}\right)=\gamma\left(t, T_{i}, T_{i+1}\right)=\frac{\delta L\left(t, T_{i}\right)}{1+\delta L\left(t, T_{i}\right)} \lambda\left(t, T_{i}\right) \quad(1 \leq i \leq N-1),
$$

with $\eta\left(t, T_{1}\right)=0$ if we are using "interpolation by daycount fractions" to extend the model to continuous tenor as in Schlögl [18]. Hence, for $1 \leq i \leq N$

$$
\begin{aligned}
F\left(T_{i}, T_{i}\right)= & F\left(t, T_{i}\right) \exp \left\{\int_{t}^{T_{i}} \sigma\left(u, T_{i}\right) \cdot d W_{T_{i}}(u)-\frac{1}{2} \int_{t}^{T_{i}}\left\|\sigma\left(u, T_{i}\right)\right\|^{2} d u\right\} \\
= & F\left(t, T_{i}\right) \exp \left\{\int_{t}^{T_{i}} \sigma\left(u, T_{i}\right) \cdot d W_{\mathbb{Q}}(u)-\frac{1}{2} \int_{t}^{T_{i}}\left\|\sigma\left(u, T_{i}\right)\right\|^{2} d u\right\} \\
& \times \exp \left\{\int_{t}^{T_{i}} \sigma\left(u, T_{i}\right)^{\top} \eta\left(u, T_{i}\right) d u\right\},
\end{aligned}
$$

where $\|\cdot\|$ denotes the inner product norm. Furthermore, futures follow the general relation

$$
G(t, T)=\mathbb{E}_{\mathbb{Q}}\left[S(T) \mid \mathcal{F}_{t}\right]
$$


see e.g. Miltersen and Schwartz [14] and Cox, Ingersoll and Ross [6], where $S(t)$ is the spot price, which satisfies by no-arbitrage constraints $S(t)=F(t, t)=G(t, t)$ for all $t$. Putting these relations together, the futures in (19) can be expressed as

$$
\begin{aligned}
G\left(t, T_{i}\right)= & \mathbb{E}_{\mathbb{Q}}\left[F\left(T_{i}, T_{i}\right) \mid \mathcal{F}_{t}\right] \\
= & F\left(t, T_{i}\right) \mathbb{E}_{\mathbb{Q}}\left[\exp \left\{\int_{t}^{T_{i}} \sigma\left(u, T_{i}\right) \cdot d W_{\mathbb{Q}}(u)-\frac{1}{2} \int_{t}^{T_{i}}\left\|\sigma\left(u, T_{i}\right)\right\|^{2} d u\right\}\right. \\
& \left.\quad \times \exp \left\{\int_{t}^{T_{i}} \sigma\left(u, T_{i}\right)^{\top} \eta\left(u, T_{i}\right) d u\right\} \mid \mathcal{F}_{t}\right],
\end{aligned}
$$

and is obviously not log-normally distributed, since $\eta\left(\cdot, T_{i}\right)$ depends on the forward interest rates. The difficulty here is to calculate an expectation of a random process value under a measure, for which the process is not a martingale. Similar techniques as utilised for convexity correction in interest rate theory can be applied in order to obtain an expression under the expectation operator, which is a log-normally distributed random variable with respect to the $\mathbb{Q}$-measure. ${ }^{4}$ A simple and widely used way is to make the second term in the expectation deterministic by "freezing" the level-dependence of the $\eta\left(\cdot, T_{i}\right)$ with respect to the currently observed forward curve, i.e. defining $\bar{\eta}\left(\cdot, T_{i}\right)$ by

$$
\bar{\eta}\left(t, T_{i+1}\right)-\bar{\eta}\left(t, T_{i}\right)=\frac{\delta L\left(0, T_{i}\right)}{1+\delta L\left(0, T_{i}\right)} \lambda\left(t, T_{i}\right) .
$$

Then all volatility terms in (20) are deterministic and we have

$$
G\left(t, T_{i}\right)=F\left(t, T_{i}\right) \exp \left\{\int_{t}^{T_{i}} \sigma\left(u, T_{i}\right)^{\top} \bar{\eta}\left(u, T_{i}\right) d u\right\} \quad(1 \leq i \leq N)
$$

In Appendix A we show how to compute the integral in (22) when the volatility functions $\sigma(t, T)$ and $\bar{\eta}(t, T)$ are piecewise constant.

Remark 4.1 Based on the convexity correction (22), the identity of the correlation matrices for forward and futures returns follows immediately.

\section{$5 \quad$ Merging Interest Rate and Commodity Calibrations}

So far the interest rate market and the commodity market have been considered separately, with exception of the convexity correction in (22) that involved the forward interest rate volatility. In this section we focus on linking both volatility matrices for building a joint Commodity LMM. The linkage is controlled by the correlation matrix between interest rate and commodity forwards. The whole correlation matrix, i.e. for any pair of forwards or forward rates within the set of commodity forwards and forward interest rates, is assumed to be constant over time. Further,

\footnotetext{
${ }^{4}$ See e.g. Pelsser [17], Chapter 11, for details on convexity correction.
} 
we assume that the calendar time discretization, for which the matrices of piecewise constant volatility for commodities and interest rates have been calibrated, coincide. The forward time discretization may differ.

The method to be described has to be applied separately for every calendar time, which is therefore fixed to some $t_{i}$ in the following. The same techniques as applied for factor reduction in context of Section 3 will be employed here. We add to the notation of the aggregate volatility matrix $V$ from Section 3 the subscript $C$ for "commodity" in order to distinguish it from its interest rate equivalent, which is subscripted by $I$. Further, we decompose the volatility matrices $V_{C}$ (obtained from the calibration in Section 3) and $V_{I}$ (obtained from LMM calibration, e.g. by the method described in Pedersen [16]) to matrices

$$
U_{C}=\left(u_{j, k}^{C}\right)_{1 \leq j \leq n_{f}, 1 \leq k \leq d_{C}} \text { and } U_{I}=\left(u_{j, k}^{I}\right)_{1 \leq j \leq m_{f}, 1 \leq k \leq d_{I}},
$$

where $m_{f}$ is the number of interest forward rates and $d_{C}$ and $d_{I}$ are the number of factors with significant eigenvalues for commodity forwards and interest forward rates, respectively. The columns of each matrix correspond to the stochastic factors and the rows to different forward times. Moreover, each column vector is orthogonal to the other column vectors within the same matrix.

We start to link both calibrations by choosing a parameter $d \geq d_{C}, d_{I}$, which determines the number of relevant factors for both, commodities and interest rates. $d$ will be also the dimension of the vector of Brownian motions in the joint Commodity LMM. If $d$ is chosen to be greater than $d_{C}$ or $d_{I}$, respectively, the corresponding matrices $U_{C} \in \mathbb{R}^{n_{f} \times d_{C}}$ and $U_{I} \in \mathbb{R}^{m_{f} \times d_{I}}$ have to be enlarged to matrices $U_{C} \in \mathbb{R}^{n_{f} \times d}$ and $U_{I} \in \mathbb{R}^{m_{f} \times d}$, simply by adding zero columns at the end. The forward interest rates now depend only on factors that relate to non-zero columns, but it is different for commodity forwards. Our fitting procedure, presented below, subsequently modifies $U_{C}$ and therefore determines which factors will have impact solely on the commodity forwards, which will have no impact on the commodity forwards and which will contribute to both, commodity forwards and forward interest rates, and therefore to the cross correlation.

The separate calibrations for commodities and interest rates are merged by matching the model intrinsic cross-covariance matrix $U_{C} U_{I}^{\top}$ with the cross-covariance matrix calculated from the exogenously given cross-correlation matrix $C_{C I}$,

$$
\Sigma_{C I}^{\text {target }}=\sqrt{\operatorname{diag}\left\{v_{C i} v_{C i}^{\top}\right\} \operatorname{diag}\left\{v_{I i} v_{I i}^{\top}\right\}^{\top}} \odot C_{C I},
$$

where diag (applied to a matrix) returns the diagonal of the matrix as column vector and the square root has to be applied component-wise. As in Section $3, v_{C i}$ and $v_{I i}$ denote the $i$ th row (corresponding to calendar time $t_{i}$ ) of $V_{C}$ and $V_{I}$, respectively, written as column vectors. In order to achieve $U_{C} U_{I}^{\top} \approx \Sigma_{C I}^{\text {target }}$ we exploit the property of multivariate normal distributed random variables to be invariant to orthonormal rotations. That means we have to find a matrix $Q$, satisfying $Q Q^{\top}=I_{d}$ (where $I_{d}$ denotes the $d \times d$-identity matrix) which minimizes

$$
r_{1}=\zeta_{1}\left\|\Sigma_{C I}^{\text {target }}-U_{C} Q U_{I}^{\top}\right\|
$$


with respect to some matrix norm, e.g. the Frobenius norm. The weight factor $\zeta_{1}$ may be necessary when further constraints have to be controlled by the loss function as well, as will be discussed below. Alternatively, it is also possible to define the cross-correlation matrix to be the target matrix and minimize

$$
r_{1}=\zeta_{1}\left\|C_{C I}-\left(U_{C} Q U_{I}^{\top}\right) \odot\left(\operatorname{diag}\left\{v_{C i} v_{C i}^{\top}\right\} \operatorname{diag}\left\{v_{I i} v_{I i}^{\top}\right\}^{\top}\right)^{-\frac{1}{2}}\right\|,
$$

subject to the same orthonormality constraint for $Q$. The exponent $-\frac{1}{2}$ has to be applied component-wise.

As with calibration, the optimisation for obtaining a $Q$ can be performed by any non-linear optimisation procedure that allows for non-linear constraints, or by non-linear least-squares algorithms like Levenberg/Marquardt, in which the distance of $Q Q^{\top}$ to the identity matrix $I_{d}$ is again controlled by a matrix norm,

$$
r_{2}=\zeta_{2}\left\|Q Q^{\top}-I_{d}\right\|,
$$

ensuring that $Q$ is as close as possible to a valid orthonormal rotation preserving the original interest rate and commodity calibrations.

Remark 5.1 The cross-correlations between commodity forwards and interest rate forwards are much lower than the correlations within the asset classes, and estimating from historical data appears to be much more volatile for the cross-correlations than for the correlations within the asset classes. For example, the structure of the cross-correlation matrix between WTI Crude Oil forwards and USD interest rate forwards in Figure 7 of Section 6 can hardly be explained by obvious rationales. Therefore, in practice one might wish to specify a flat cross-correlation founded on particular market views. This can be realized in a straightforward manner in our approach but the example in Section 6 below shows that it is also possible to adequately match a more complicated cross-correlation structure. In the end, there is a trade-off between quality of the cross-correlation fit and the number of stochastic factors in the model. In view of computational efficiency and model parsimony a limited number of factors is desirable and perhaps more important than exactly fitting a given cross-correlation structure.

Remark 5.2 In our approach the basis transformation $Q$ applies to the commodity volatility matrix $U_{C}$. Alternatively, on could choose the interest rate volatility matrix $U_{I}$ for transformation. This would not change criteria $r_{1}$ and $r_{2}$, because $Q$ can also be interpreted as $Q^{\top}$ with inverse $Q$, and the transformed model covariance matrix would read as $U_{C}\left(U_{I} Q\right)^{\top}$.

The determination of the basis transformation matrices concludes the calibration of the model, if the market instruments are forwards and options on forwards. However, if the calibration is carried out for futures and options on futures, the model option prices would change with any non-trivial basis transformation, since the forward prices are modified by means of the convexity correction (22). 
This implies that the whole calibration process has to be iterated by, firstly, refitting the volatility matrix $V_{C}$, where in each step of the optimisation the corresponding basis transformation matrix $Q$ has to be multiplied to the volatility decomposition $U_{C}$ (for each calendar time) before the model call prices and the value of the loss function are computed. Secondly, for the refitted $V_{C}$ and $U_{C}$ new basis transformations have to be determined in order to still match the crosscorrelations.

Both steps, the fitting of $V_{C}$ and $Q$, have to be iterated until a sufficient smoothness and quality of fit subject to the market option prices and the exogenously given cross-correlation is reached. For the real data example of the following section this happens already after 3 iterations in the case with 6 stochastic factors.

For clarity we summarise the steps of the whole calibration process to futures and options on futures:

\section{Preliminary calculations applied to the LMM calibration outcome.}

1. Computations for each calendar time $t_{i}\left(1 \leq i \leq n_{I}\right)$ :

(a) Computation of the covariance matrix $\Sigma_{I i}$ as in (16).

(b) Decomposition of $\Sigma_{i}$ into $U_{I i}$ using PCA in the way described at the end of Section 3.

II. Iteration until a sufficient quality of fit based on the criteria on matching market option prices, smoothness and cross-correlation is reached.

1. Calibration of $V_{C}$.

Minimisation of the penalty function given in (d):

(a) Computations for each calendar time $t_{i}\left(1 \leq i \leq n_{C}\right)$ :

i. Computation of the covariance matrix $\Sigma_{C i}$ by (16).

ii. Decomposition of $\Sigma_{C i}$ into $U_{C i}$ using PCA as described at the end of Section 3.

iii. Multiplication with the basis transform $Q_{i}$ resulting from the previous iteration, $U_{C i} Q_{i}$; ( $Q_{i}$ is identity matrix in the very first iteration).

(b) Computation of forward prices from market futures prices using (22).

(c) Computation of model prices for options on forwards using (17) and the Black formula.

(d) Calculation of the loss value $q+s_{1}+s_{2}+s_{3}$ as defined in Section 3 .

\section{Fit of the basis transformations.}

Fitting $\left\{Q_{i}\right\}_{1 \leq i \leq n_{c}}$ subject to the penalty function $r_{1}+r_{2}$ as described in this section. 
Remark 5.3 The number of factors $d_{C}$ can affect the number of iterations required for the calibration process to converge satisfactorily. Equations (21) and (22) demonstrate that if the cross-correlation is zero, futures and forward prices coincide. On the other hand, the first fit of the volatility matrices $U_{C}$ is made without any consideration of cross-correlation. Hence, (unintentionally) assigning in the computation of $U_{C}$ the most contributing eigenvalues and eigenvectors of $V_{C}$ to those stochastic factors of the d-dimensional Brownian motion, which also represent strong $V_{I}$ contributions, would generate a rather high cross-correlation in the first calibration step at the end of step II.1 above. In this case the forward curve would tend to depart from the futures curve. However, if the exogenous cross-correlation has a low level, the basis transformation would modify the volatility matrix $U_{C}$ in a way that not only reduces the high model cross-correlation generated in the first calibration step, but also returns the forward curve in the direction of the futures curve by reducing the magnitude of the convexity correction.

An iteration of the procedure (as described above) will typically (in all real data scenarios that we have considered) force the objective variables $V_{C}$ and $Q$ to a balanced state with adequate calibration results. As demonstrated in the following section, the convergence is much slower if $d_{C}$ is large relative to $d_{I}$, since a large $d_{C}$ allows for more de-correlation than a small one.

Finally, in the last iteration of the procedure described above we end up with decomposed volatility matrices $U_{C}$ and transformation matrices $Q$ for each calendar time, and assembling these resulting $n_{c}$-many matrices $U_{C} Q$ and $U_{I}$ to 3 -dimensional arrays $\Lambda_{C} \in \mathbb{R}^{n_{c} \times n_{f} \times d}$ and $\Lambda_{I} \in \mathbb{R}^{n_{c} \times m_{f} \times d}$ the calibration of the hybrid Commodity LMM is finished ${ }^{5}$. Note, that $\Lambda_{I}$ basically consists of piecewise constant forward interest rate volatilities $\lambda(t, T)$ as occurring in (6), obtained from the separate LMM calibration, at most modified by some interpolation on these calibrated volatilities in order to obtain $\Lambda_{I} . \Lambda_{C}$ is the result of the calibration method as described in the previous sections, together with the transformation presented in this section in order to match cross correlations.

This allows us to write the dynamics of the hybrid Commodity LMM as follows. Let $W$ be a $d$-dimensional Brownian motion and denote by $\lambda_{i, j}^{I}$. and $\lambda_{i, j}^{C}$, the $d$-dimensional vectors in $\Lambda_{I}$ and $\Lambda_{C}$ of volatilities for calendar times $t \in\left[t_{i-1}, t_{i}\right)$ and times to maturity $x \in\left[x_{j-1}, x_{j}\right)$. Then, the dynamics of the forward interest rates $L(t, T)$ as given in (6) can be written as

$$
d L(t, T)=L(t, T) \lambda_{i, j, \cdot}^{I} \cdot d W_{T}(t),
$$

and the dynamics of the commodity forwards $F(t, T)$ as given in (9) as

$$
d F(t, T)=F(t, T) \lambda_{i, j, \cdot}^{C} \cdot d W_{T}(t),
$$

for all maturity times $T$ satisfying $T-t \in\left[x_{j-1}, x_{j}\right.$ ) and all calendar times $t_{i-1} \leq t<t_{i}$ (for some $1 \leq i \leq n_{c}$ and $1 \leq j \leq m_{f}$ or $1 \leq j \leq n_{f}$, respectively).

\footnotetext{
${ }^{5}$ Since we had to equalise the calendar time discretisation for commodities and interest rates in order to be able to fit a transformation matrix $Q$, the first dimension of $\Lambda_{I}$ is of size $n_{c}$. The choice to adapt the forward interest rate discretisation of calendar time to the commodity discretisation is arbitrary and any other discretisation could be used.
} 

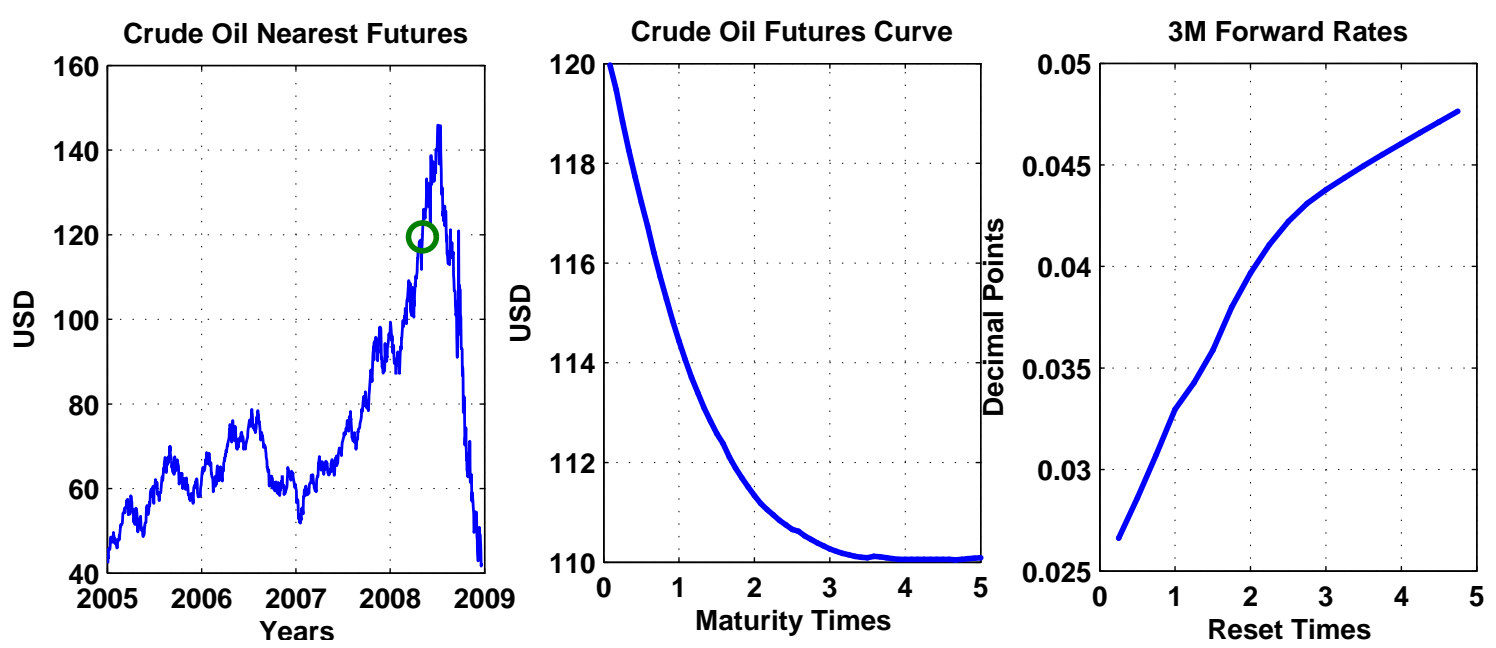

Figure 1: The commodity and interest market for calibration date May, $5^{\text {th }}$ 2008. Left: The WTI Crude Oil nearest futures between 2005 and end of 2008. The circle indicates the calibration date. Middle: The futures curve as seen at calibration date with maturities up to five years. Right: The 3-month USD forward rates for reset dates (expiries) between 3 months and 4 years and 9 months.

\section{Real Data Example}

We demonstrate the performance and applicability of the Commodity LIBOR Market Model by calibrating it to real data. ${ }^{6}$ We have chosen the May, $5^{\text {th }} 2008$ for calibration and WTI Crude Oil as commodity, hence, the US Dollar (USD) forward rate as interest rate. As can be seen in Figure 1, the nearest WTI Crude Oil future price of 119.97 USD was not too far from its peak in July 2008. The futures curve is in 'backwardation' and covers a rather large range of about 10 USD within the first five years of maturity. The 3-month forward rates show a less extreme pattern than the commodity futures. An application to 2009 data produced comparable results to those presented in this example regarding the fit to commodity and fixed income market data, but at the cost of a slightly rougher forward interest rate volatility surface.

The calibration of the (classical) LMM was done as proposed by Pedersen in [16]. Figure 2 shows the resulting volatility surface and the correlation matrix as used for calibration, which has been historically estimated from the time series of forward rates covering 3 months before May, $5^{\text {th }}$ 2008. Caplets, caps and swaptions were used for calibration and the fit to market prices is quite good, as Figure 3 demonstrates. Note, that we have to employ the forward interest rate curve up to 6 years forward time, in order to calculate the convexity correction for a commodity volatility surface with 3 years calendar and 3 years forward time. The calibration of the WTI

${ }^{6}$ The data was taken from the SuperDerivatives platforms SD-IR and SD-CM. 

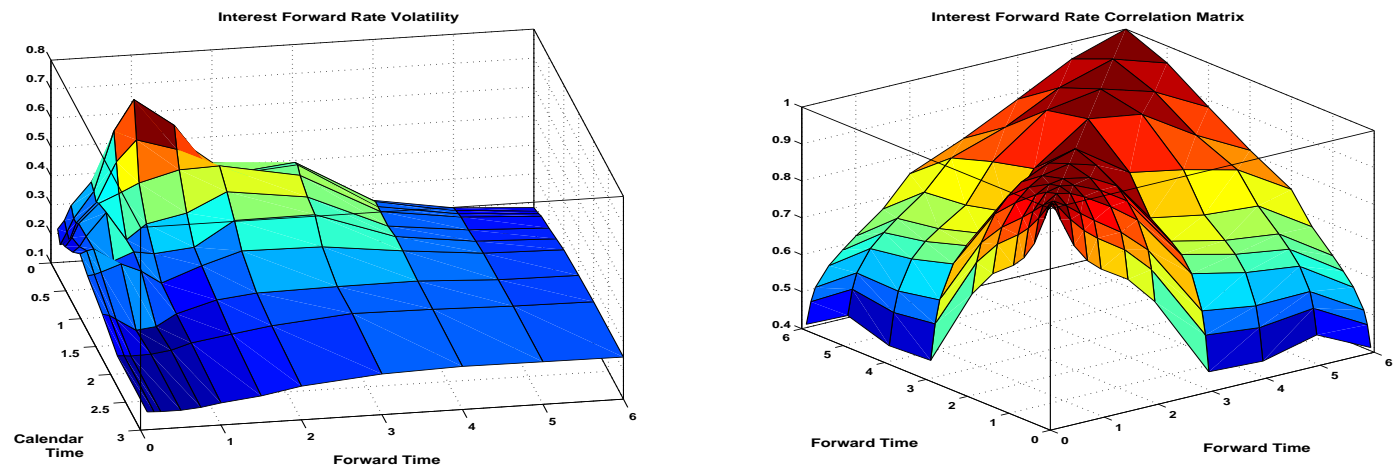

Figure 2: Left: The calibrated volatility matrix. Right: The historically estimated correlation matrix as used for calibration.
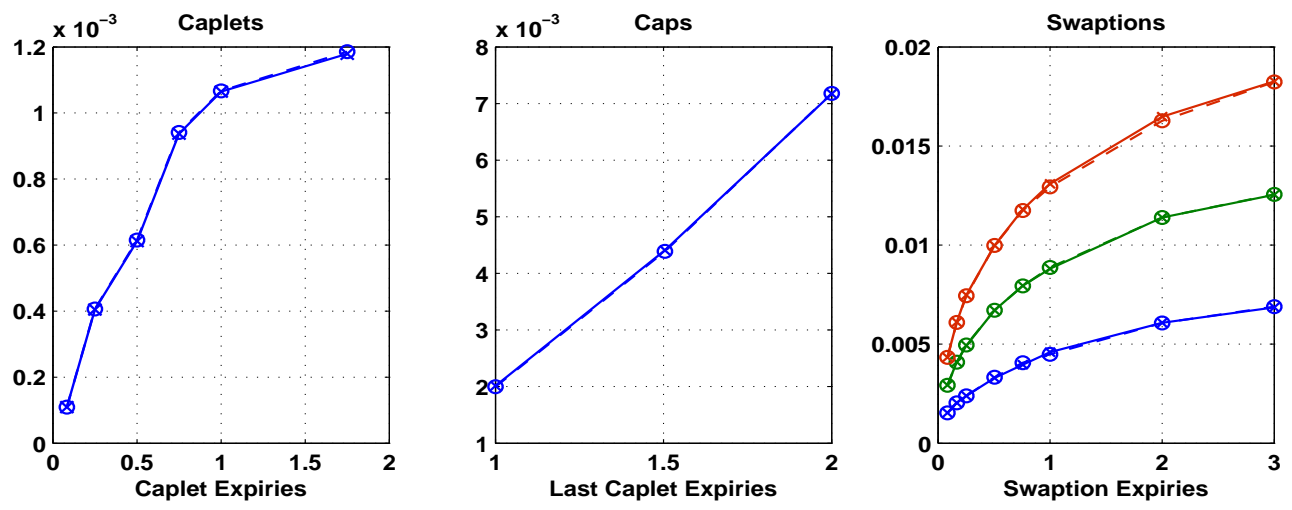

Figure 3: Market prices versus model prices. The market prices are represented by crosses connected by solid lines, the model prices by circles connected by dashed lines. Left: Caplets. Middle: Caps. Right: Swaptions with 1 year, 2 year and 3 year tenors (from bottom to top).

Crude Oil futures was achieved by the method described in Section 3. The market instruments are futures and options on futures traded on the New York Mercantile Exchange. Figure 4 shows the calibrated volatility surface and the historically estimated correlation matrix using 3 months of futures prices before the calibration date. Calendar and forward times go out to 3 years, and although on the exchange futures with expiries in every month are traded, we chose the calendar and forward time vectors to be unequally spaced (while still calibrating to all traded instruments), with 1 month difference up to 1 year, 2.4 months difference between 1 and 2 years and 6 months difference between 2 and 3 years. This speeds up the calibration without losing much structure in the volatility surface, since the market views futures with long maturity to have almost flat volatility. For weighting in the calibration objective function we have chosen $\eta_{q}=1$ 

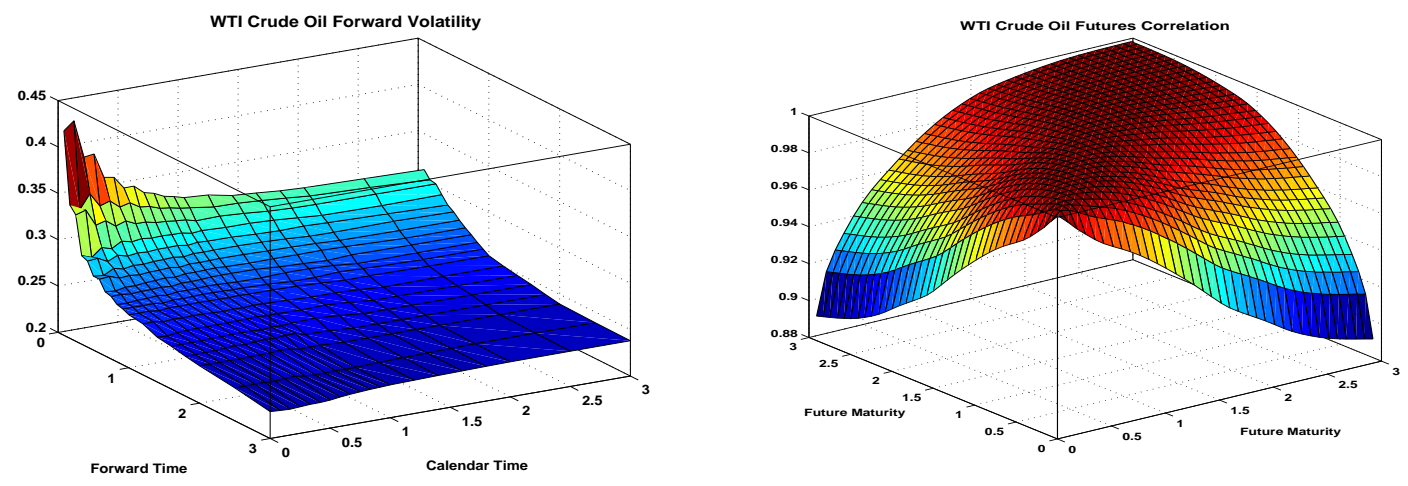

Figure 4: Left: The calibrated commodity volatility surface. Right: The historically estimated correlation matrix as used for calibration.
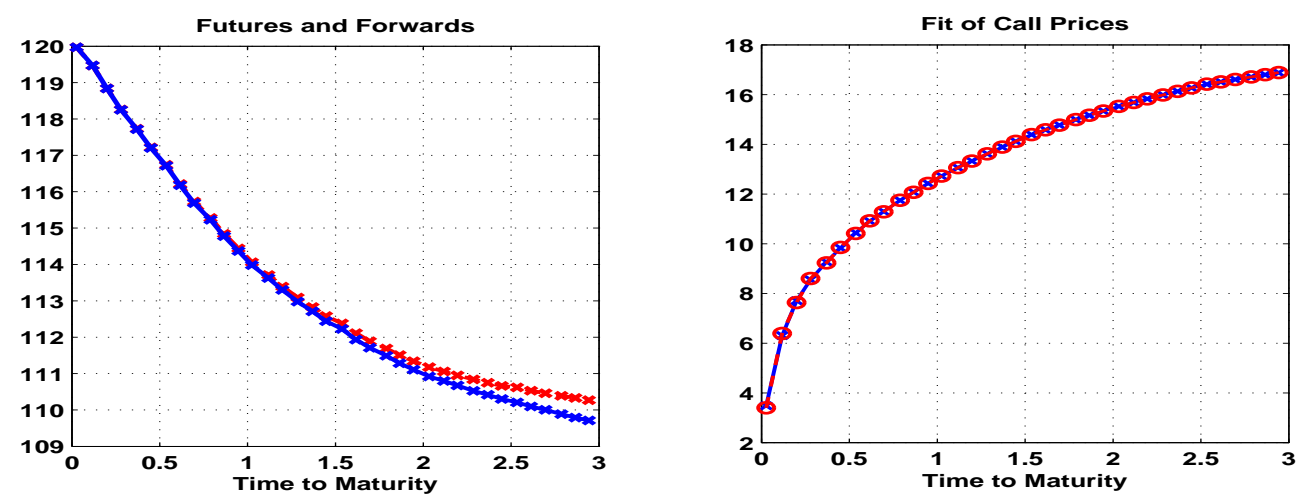

Figure 5: Left: The differences between futures (dashed red line) and forwards (solid blue line) as calculated from the convexity correction. Right: Fit of model prices (red circles connected by dashed line) to market prices (blue crosses connected by solid line).

(fit to call prices), $\eta_{1}=0.1$ (time homogeneity, i.e. smoothness in calendar time direction), $\eta_{3}=0.01$ (smoothness in forward time direction) and $\eta_{2}=0.1$ (decreasing monotonicity in forward time direction). As initial guess we have interpolated the implied volatilities to the forward time grid for the first calendar time $t_{1}$ and then used constant extrapolation to all other calendar times.

The model fit to the commodity call prices is very good, as illustrated in the right graph of Figure 5. The left graph shows the difference between futures and forwards as calculated by the convexity correction.

In Figure 6 particular slices of the forward volatility structure are examined. The left graph shows the term structure of volatilities for certain calendar times, whereas the right graph 

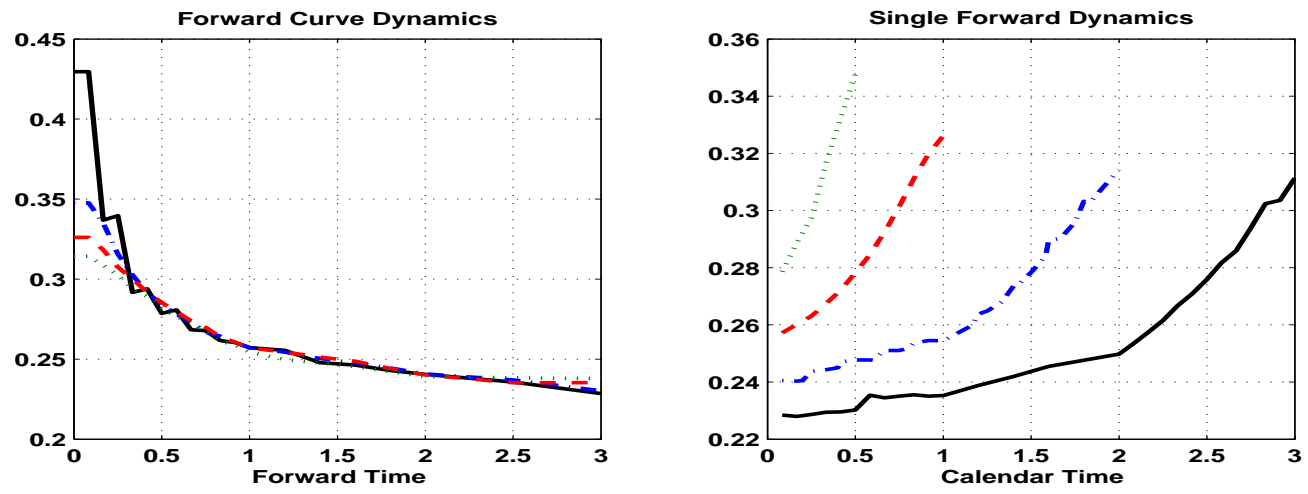

Figure 6: Left: Forward volatility structures at different calendar times (solid black $=0$ (calibration date), dotted-dashed blue $=6$ month, dashed red $=1$ year, dotted green $=2$ years). Right: Evolution of forward volatilities over lifetime (solid black = forward with 3 years maturity, dashed-dotted blue $=2$ years, dashed red $=1$ year, dotted green $=6$ month) .

illustrates the evolution of volatilities over the lifetime of different forwards.

Finally, we link both separately calibrated volatility matrices to one set of stochastic factors. Table 1 shows how much of the overall variance, i.e. of the sum of variances over all factors, can be explained by the leading factors, when the factors are sorted according to decreasing contribution to total variance of the commodity forwards. The first two factors already account for more than $99 \%$ of the overall variance, hence a reasonable choice would be $d_{C}=2$. Since Pedersen's calibration method for the LMM part of the model already includes a spectral decomposition of the interest forward rates covariance matrix, it is not meaningful to do it again here, because the number of factors with reasonably large contribution should be the same as the number of factors chosen for the LMM calibration. If it is necessary to interpolate the forward interest rate volatility matrix in order to match the calendar times of the commodity volatility matrix, the forward rate covariance matrix will change and, hence, eigenvalue decompositions of the calendar time adjusted covariance matrices yield different results than an eigenvalue decompositions of the original covariance matrices as used for calibration. However, these differences should not be substantial as long as the calibrated volatility matrix is sufficiently smooth in calendar time. For the LMM calibration we have chosen the number of factors to be $d_{I}=4$, which again covered about $99 \%$ of the overall variance.

We now have to determine the parameters for the final cross-correlation fit. First, we choose the total number of factors in the joint model to be $d=6$ and allow the commodity volatility matrix to disperse over all six factors, whereas the interest rate volatilities should remain on the first four factors, since we apply the basis transform to the commodity volatilities (see also Remark 5.2). Figure 7 demonstrates the result of the transformation. The left graph shows two surfaces, the exogenously given target cross-correlation matrix (as calculated from 


\begin{tabular}{ccccccccc}
\hline No. of Factors & 1 & 2 & 3 & 4 & 5 & 6 & $\ldots$ & 19 \\
\hline $\begin{array}{c}\text { Percentage of } \\
\text { Overall Variance }\end{array}$ & 98.138 & 99.835 & 99.957 & 99.979 & 99.988 & 99.992 & $\ldots$ & 100 \\
\hline
\end{tabular}

Table 1: Results of the eigenvalue decomposition of the commodity forward covariance matrix for the first calendar time $t_{1}$. The maximum number of factors coincides with the number of forward times greater zero. The second row shows for the $i$ th factor the percentage of overall variance that can be generated by the first $i$ factors.

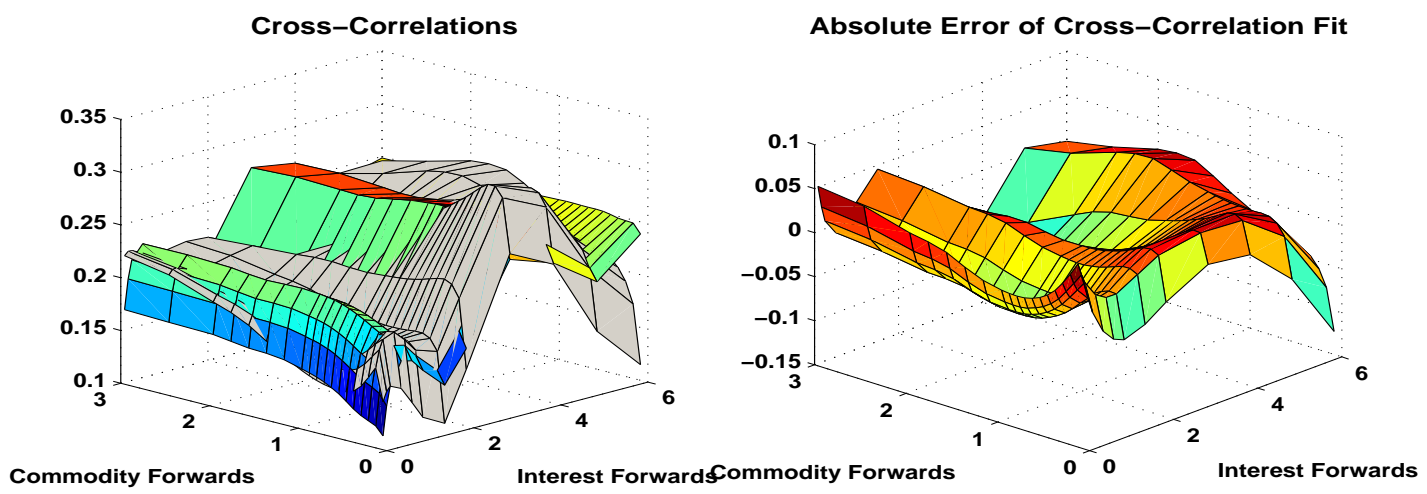

Figure 7: The cross-correlation fit with $d_{C}=d=6$. Left: The target cross-correlation matrix (colored) as estimated from historical futures returns and the cross-correlation matrix for the calendar time that fitted worst (gray). Right: Differences between target and fitted crosscorrelations.

the historical time series covering 3 months before the calibration time) and the transformed correlation matrix for the particular calendar time that fitted worst with respect to the Frobenius norm. The right graph illustrates the absolute differences between target cross-correlation and the fitted cross correlation matrix of the left graph. For optimisation we applied a non-linear Levenberg/Marquardt algorithm with scale parameters $\zeta_{1}=1$ (quality of fit) and $\zeta_{2}=10$ (orthonormality of transformation matrix). Without the application of a basis transformation, i.e. by optimising only subject to the quality of fit and smoothness criteria, the cross-correlation ranges between 0.5 and 1 for all commodity and interest rate forwards over all calendar times.

By construction, the aggregated commodity volatility matrix, as shown in the left graph of Figure 4, does not change. How the decomposed factors change is illustrated in Figure 8. The left graph shows only the first four factors of the original calibration without considering crosscorrelations, since the other are very close to zero. The right graph demonstrates how the basis transformation spreads the contribution of the relevant factors of the original calibration to all available factors in order to match the cross-correlation coefficients. 

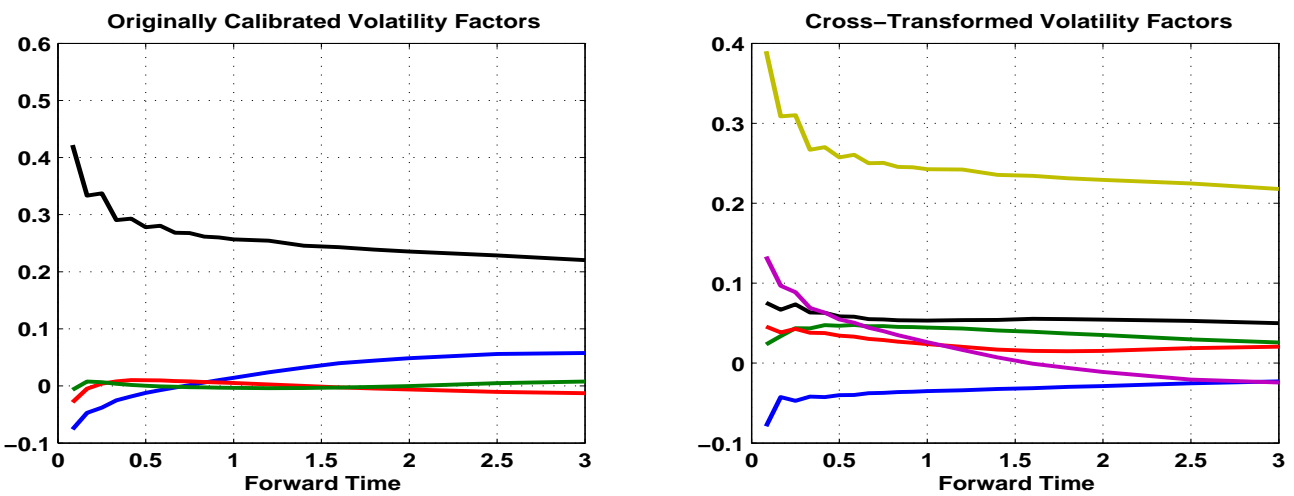

Figure 8: The factorised commodity volatilities of the first calendar time $t_{1}$. Left: The first four factors from the initial calibration, without considering cross relations. The $5^{\text {th }}$ and $6^{\text {th }}$ factors are not shown since they are almost zero. Right: All six factors after applying the basis transformation in order to match the cross correlations. The $5^{\text {th }}$ and $6^{\text {th }}$ factors are represented by those two lines that have the largest value at forward time closest to zero.
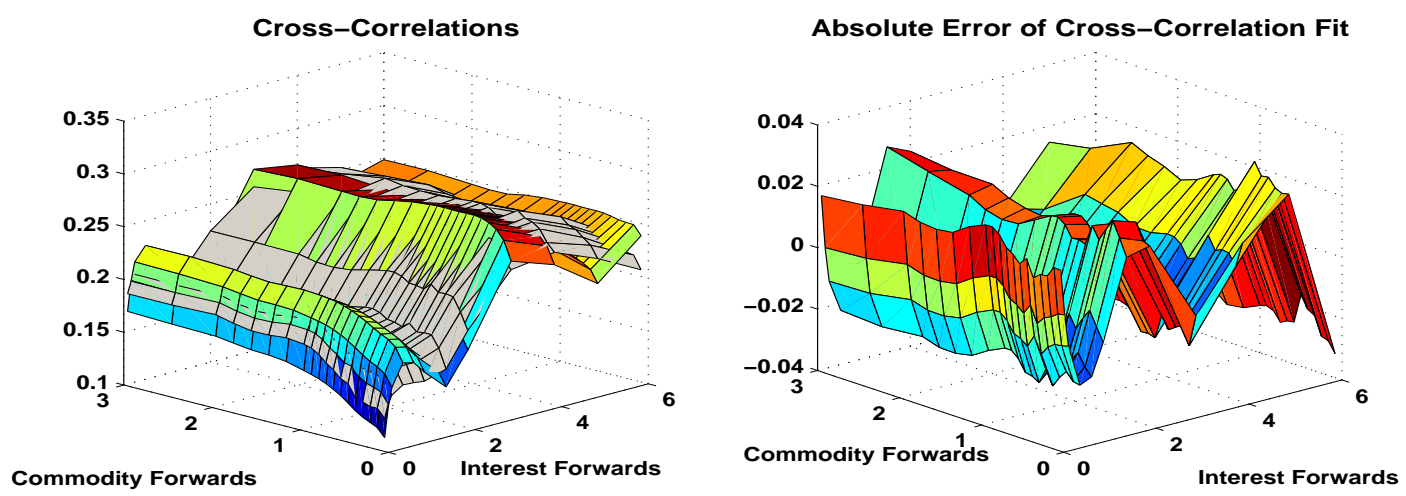

Figure 9: The cross-correlation fit with $d_{C}=d=12$. Left: The target cross-correlation matrix (colored) as estimated from historical futures returns and the cross-correlation matrix for the calendar time that fitted worst (gray). Right: Differences between target and fitted cross-correlations.

The quality of the cross-correlation fit can be substantially improved by increasing $d_{C}$ and hence $d$. Allowing for 6 more factors, i.e. setting $d_{C}=d=12$ results in cross-correlation fits as shown in Figure 9.

The choice of $d_{C}-d_{I}=8$ independent factors for the commodity forwards allows the fitted basis transformation matrices in the very first iteration to have a strong de-correlation effect. This is especially true compared to the high cross-correlation (between 0.5 and 1.0) obtained from 


\begin{tabular}{|c|ccccccc|}
\hline \multirow{5}{*}{$d_{C}=6$} & Iteration & 1 & 2 & 3 & 4 & 5 & 6 \\
\cline { 2 - 8 } & $\ell_{1}$ & 0.0261 & 0.0265 & 0.0263 & - & - & - \\
& $\min \left\{\ell_{2 i}\right\}$ & 0.2373 & 0.2409 & 0.2414 & - & - & - \\
& $\max \left\{\ell_{2 i}\right\}$ & 0.3544 & 0.3922 & 0.3972 & - & - & - \\
\hline \multirow{5}{*}{$d_{C}=12$} & Iteration & 1 & 2 & 3 & 4 & 5 & 6 \\
\cline { 2 - 8 } & $\ell_{1}$ & 0.0261 & 0.9852 & 0.9550 & 0.7856 & 0.7828 & 0.6816 \\
& $\min \left\{\ell_{2 i}\right\}$ & 0.2004 & 0.2005 & 0.2005 & 0.2000 & 0.2000 & 0.2000 \\
& $\max \left\{\ell_{2 i}\right\}$ & 0.2551 & 0.2636 & 0.2543 & 0.2879 & 0.2868 & 0.2539 \\
\cline { 2 - 9 } & Iteration & 7 & 8 & 9 & 10 & 11 & 12 \\
\cline { 2 - 8 } & $\ell_{1}$ & 0.6367 & 0.6301 & 0.6327 & 0.5090 & 0.4146 & 0.3992 \\
& $\min \left\{\ell_{2 i}\right\}$ & 0.2001 & 0.2000 & 0.2000 & 0.2001 & 0.2012 & 0.2012 \\
& $\max \left\{\ell_{2 i}\right\}$ & 0.2538 & 0.2537 & 0.2537 & 0.2538 & 0.2539 & 0.2537 \\
\hline
\end{tabular}

Table 2: Calibration with $d_{C}=6$ and 3 iterations compared to $d_{C}=12$ and 12 iterations. The value $\ell_{1}$ denotes the final loss function value of the $V_{C}$ optimization in step II.1. The value $\ell_{2 i}$ denotes the Frobenius norm of the difference between model and target cross-correlation matrix for calendar time $t_{i}$ as induced by the fitted $Q_{i}$ in step II.2.

fitting $V_{C}$ without any consideration of cross-correlation in step II.1 of the very first iteration. Table 2 demonstrates that for $d_{C}=12$ more iterations are required to find a balance for the fits of $V_{C}$ and the cross-correlation matrix.

Figure 10 shows the forward prices and the fit to the market call prices for both calibrations. Whereas the forwards almost coincide, the fit to market call prices seems to be worse in the case of $d_{C}=12$, especially for options with long maturities. The left graph of Figure 11, which is a detail of the right graph in Figure 10, confirms this and explains the larger loss value $\ell_{1}$. The smoothness of the volatility matrix $V_{C}$ is lower for $d_{C}=12$ as is demonstrated in the right graph of Figure 11. This further contributes to a higher $\ell_{1}$.

\section{$7 \quad$ Pricing Spread Options}

This section examines an approximation approach in order to price spread options on forwards and futures following Kirk [10]. Spread options can be used to hedge the risk of roll over costs, i.e. the price difference between two futures or forwards with different maturities. Moreover, spread options depend on the correlation and the difference between the volatilities of the involved futures or forwards, hence, can be employed to reduce risk generated by these parameters. We will first consider forwards and then sketch an analogous derivation for futures.

Let $T_{i}, T_{j}$ denote two forward maturities with $T_{i}<T_{j}$ and we set the option expiration time to $T_{i}$. According to market practice, the spread is defined by $S_{i, j}(t)=F\left(t, T_{j}\right)-F\left(t, T_{i}\right)$ and the spread call option value is given by

$$
C_{\text {Spread }}^{\mathrm{Fwd}}\left(0, T_{i}, T_{j}, K\right)=\mathbb{E}_{\mathbb{Q}}\left[D\left(T_{i}\right)\left(S_{i, j}\left(T_{i}\right)-K\right)^{+} \mid \mathcal{F}_{0}\right]
$$



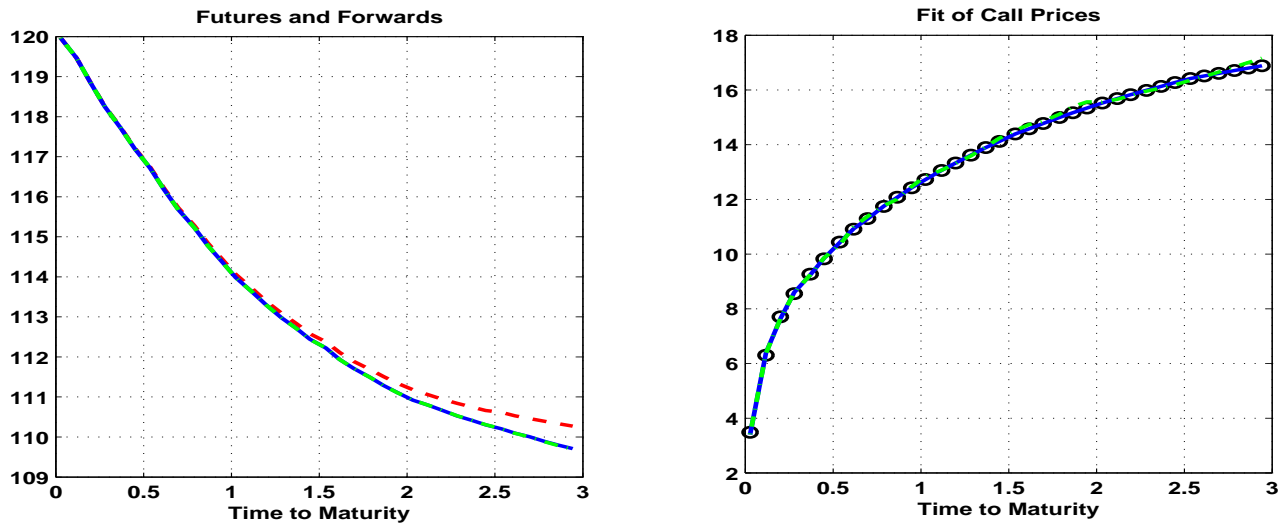

Figure 10: Comparison of the calibration results for $d_{C}=6$ with 3 iterations and $d_{C}=12$ with 12 iterations. Left: The market future prices (red dashed line) and the forward prices as calibrated with $d_{C}=6$ (blue solid line) and $d_{C}=12$ (green dotted-dashed line). Right: The market call prices (black circles) and the model call prices for $d_{C}=6$ (solid blue line) and $d_{C}=12$ (green dotted-dashed line).

$$
=B\left(0, T_{i}\right) \mathbb{E}_{T_{i}}\left[\left(F\left(T_{i}, T_{j}\right)-F\left(T_{i}, T_{i}\right)-K\right)^{+} \mid \mathcal{F}_{0}\right] .
$$

From Section 2 we know that

$$
\begin{aligned}
& d F\left(t, T_{i}\right)=F\left(t, T_{i}\right) \sigma\left(t, T_{i}\right) \cdot d W_{T_{i}}(t) \\
& d F\left(t, T_{j}\right)=F\left(t, T_{j}\right) \sigma\left(t, T_{j}\right)^{\top}\left(\sum_{\ell=i}^{j-1} \frac{\delta L\left(t, T_{\ell}\right)}{1+\delta L\left(t, T_{\ell}\right)} \lambda\left(t, T_{\ell}\right)\right) d t+F\left(t, T_{j}\right) \sigma\left(t, T_{j}\right) \cdot d W_{T_{i}}(t) .
\end{aligned}
$$

By "freezing" the level-dependence of the $L\left(\cdot, T_{\ell}\right)$ with respect to the currently observed forward curve as described in Section 4, both processes become geometric Brownian motions,

$$
\begin{aligned}
& d F\left(t, T_{i}\right)=F\left(t, T_{i}\right) \sigma\left(t, T_{i}\right) \cdot d W_{T_{i}}(t), \\
& d F\left(t, T_{j}\right) \approx F\left(t, T_{j}\right) \sigma\left(t, T_{j}\right)^{\top} \Gamma_{i, j-1}(t) d t+F\left(t, T_{j}\right) \sigma\left(t, T_{j}\right) \cdot d W_{T_{i}}(t),
\end{aligned}
$$

with

$$
\Gamma_{i, j-1}(t)=\sum_{\ell=i}^{j-1} \frac{\delta L\left(0, T_{\ell}\right)}{1+\delta L\left(0, T_{\ell}\right)} \lambda\left(t, T_{\ell}\right) .
$$

Kirk's [10] idea is to assume that instead of $F\left(t, T_{i}\right)$ in (26) $F\left(t, T_{i}\right)+K$ is a geometric Brownian motion with adjusted volatility,

$$
d\left(F\left(t, T_{i}\right)+K\right)=\left(F\left(t, T_{i}\right)+K\right) \frac{F\left(0, T_{i}\right)}{F\left(0, T_{i}\right)+K} \sigma\left(t, T_{i}\right) \cdot d W_{T_{i}}(t) .
$$



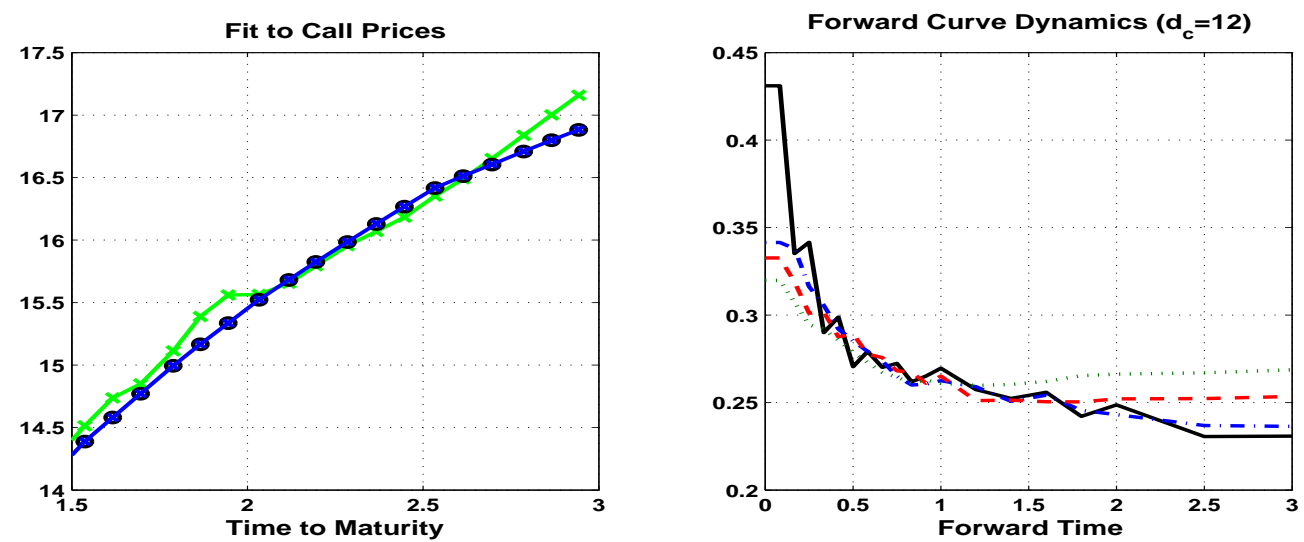

Figure 11: Comparison of the calibration results for $d_{C}=6$ with 3 iterations and $d_{C}=12$ with 12 iterations. Left: A detail of the right graph of Figure 10. The market call prices (black circles) and the model call prices for $d_{C}=6$ (solid blue line) and $d_{C}=12$ (green dotted-dashed line). Right: Forward volatilities as calibrated with $d_{C}=12$ for different calendar times (solid black $=0$ (calibration date), dotted-dashed blue $=6$ months, dashed red $=1$ year, dotted green $=2$ years). See the left graph of Figure 6 for the corresponding volatilities calibrated with parameter $d_{C}=6$.

This allows to apply Margrabe's approach [11] for options to exchange one asset for another by rewriting the payoff in $(23)$ such that

$$
C_{\text {Spread }}^{\mathrm{Fwd}}\left(0, T_{i}, T_{j}, K\right)=B\left(0, T_{i}\right) \mathbb{E}_{T_{i}}\left[\left(F\left(T_{i}, T_{i}\right)+K\right)\left(\frac{F\left(T_{i}, T_{j}\right)}{F\left(T_{i}, T_{i}\right)+K}-1\right)^{+} \mid \mathcal{F}_{0}\right] .
$$

The solution of (29) is

$$
\begin{gathered}
\frac{F\left(t, T_{i}\right)+K}{F\left(0, T_{i}\right)+K}=\exp \left\{-\frac{1}{2}\left(\frac{F\left(0, T_{i}\right)}{F\left(0, T_{i}\right)+K}\right)^{2} \int_{0}^{t} \sigma\left(s, T_{i}\right)^{\top} \sigma\left(s, T_{i}\right) d s\right. \\
\left.+\left(\frac{F\left(0, T_{i}\right)}{F\left(0, T_{i}\right)+K}\right) \int_{0}^{t} \sigma\left(s, T_{i}\right) \cdot d W_{T_{i}}(s)\right\},
\end{gathered}
$$

where the right hand side is an exponential martingale which defines a Radon-Nikodým derivative $d \hat{\mathbb{P}} / d \mathbb{P}_{T_{i}}$. Hence, the call price (30) can be written as

$$
C_{\text {Spread }}^{\mathrm{Fwd}}\left(0, T_{i}, T_{j}, K\right)=B\left(0, T_{i}\right)\left(F\left(0, T_{i}\right)+K\right) \mathbb{E}_{\hat{\mathbb{P}}}\left[\left(Y\left(T_{i}\right)-1\right)^{+} \mid \mathcal{F}_{0}\right],
$$

where the dynamics of $Y(t)=F\left(t, T_{j}\right) /\left(F\left(t, T_{i}\right)+K\right)$,

$$
d Y(t)=Y(t)\left[\sigma\left(t, T_{j}\right)^{\top} \Gamma_{i, j-1}(t) d t+\left(\sigma\left(t, T_{j}\right)-\frac{F\left(0, T_{i}\right)}{F\left(0, T_{i}\right)+K} \sigma\left(t, T_{i}\right)\right) \cdot d W_{\hat{\mathbb{P}}}(t)\right]
$$


is derived from (27) and (29) using Itô's product rule and $d W_{\hat{\mathbb{P}}}(t)=d W_{T_{i}}(t)-\frac{F\left(0, T_{i}\right)}{F\left(0, T_{i}\right)+K} \sigma\left(t, T_{i}\right) d t$. Standard techniques applied to (31) yield

$$
\begin{aligned}
& C_{\text {Spread }}^{\mathrm{Fwd}}\left(0, T_{i}, T_{j}, K\right) \\
& \quad=B\left(0, T_{i}\right)\left[F\left(0, T_{j}\right) e^{\int_{0}^{T_{i}} \sigma\left(s, T_{j}\right)^{\top} \Gamma_{i, j-1}(s) d s} N\left(d_{+}\right)-\left(F\left(0, T_{i}\right)+K\right) N\left(d_{-}\right)\right],
\end{aligned}
$$

where $N(\cdot)$ is the cumulative standard normal distribution function and

$$
\begin{aligned}
d_{ \pm} & =\frac{1}{\zeta}\left[\ln \left(\frac{F\left(0, T_{j}\right)}{F\left(0, T_{i}\right)+K}\right)+\int_{0}^{T_{i}} \sigma\left(s, T_{j}\right)^{\top} \Gamma_{i, j-1}(s) d s \pm \frac{1}{2} \zeta^{2}\right] \\
\zeta^{2} & =\int_{0}^{T_{i}}\left\|\sigma\left(s, T_{j}\right)-\frac{F\left(0, T_{i}\right)}{F\left(0, T_{i}\right)+K} \sigma\left(s, T_{i}\right)\right\|^{2} d s .
\end{aligned}
$$

Figure 12 demonstrates the applicability of this approximation approach for the market scenario as calibrated in Section 6 . The commodity forward maturities are chosen to be 1 year for $T_{i}$ and 1 year plus 3 months for $T_{j}$. Since the forward interest rate maturities do not exactly match with the commodity forward maturities, we have interpolated the commodity forward prices in order to obtain values for $F\left(\cdot, T_{i}\right)$ and $F\left(\cdot, T_{j}\right)$. For an arbitrage-free interpolation in the maturities of the forward interest rates we refer to [18]. The resulting prices for call spread options on these forwards calculated by (32) and reference prices are shown for strikes between -15 and 10 in the left graph of Figure 12. Reference prices are computed by Monte Carlo simulation using equations (24) and (25), where both commodity forwards as well as the interest forward rates $L\left(t, T_{\ell}\right)$ in $(24)$ were simulated by the same set of 500,000 Brownian motion paths for all strikes. The differences between the prices calculated by (32) and the Monte Carlo prices is shown in the upper curve of the right graph of Figure 12 and result from two approximations in the derivation of the formula: Firstly, from "freezing" the forward interest rates in (27) in order to make $\Gamma_{i, j-1}(t)$ deterministic and, secondly, from inserting the strike $K$ in the dynamics of $F\left(t, T_{i}\right)$ as done in (29). The lower curve in the right graph shows the error resulting from the second approximation only. It is the difference between the prices calculated from (32) and Monte Carlo simulated prices from the dynamics given by (25) (i.e. without "freezing" of the forward interest rates) and by (29).

We conclude this section by sketching the derivation of the analogous spread call options formula for futures instead of forwards. From the forward dynamics (26) and relation (22) we get for futures the general dynamics

$$
d G\left(t, T_{k}\right)=-G\left(t, T_{k}\right) \sigma\left(t, T_{k}\right)^{\top} \bar{\eta}\left(t, T_{k}\right) d t+G\left(t, T_{k}\right) \sigma\left(t, T_{k}\right) \cdot d W_{T_{k}}(t) \quad(1 \leq k \leq N) .
$$



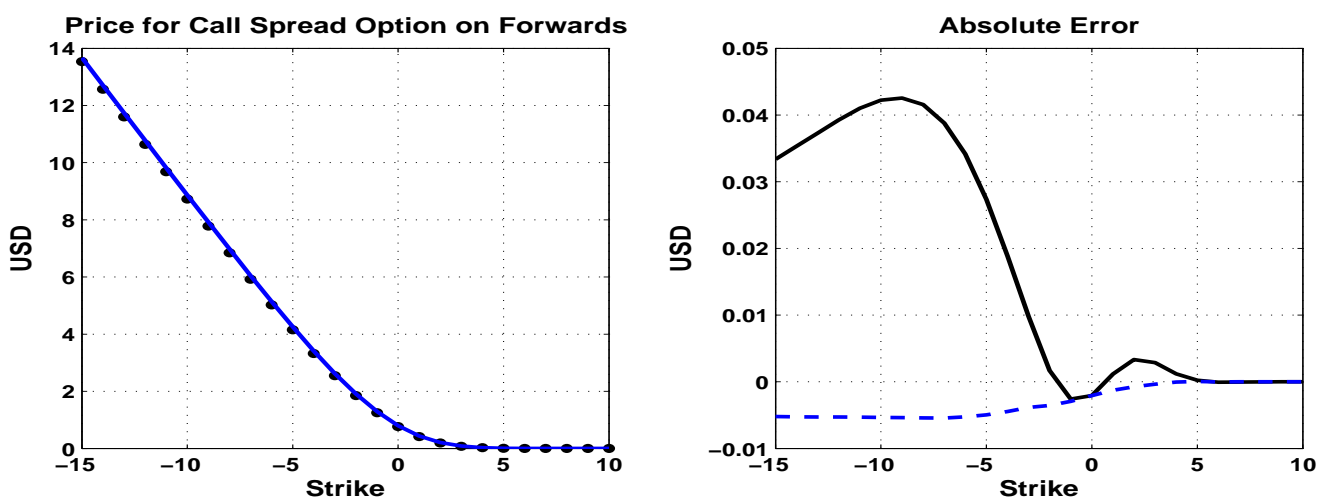

Figure 12: Call spread options on forwards for the market as calibrated in Section 6. The maturities for the commodity forwards are 1 year and 1 year plus 3 months. Left: The black dots show Monte Carlo prices with simulated commodity forwards and simulated interest forward rates (i.e. no "freezing"). The blue solid line indicates the prices calculated using (32). Right: The black solid line shows the absolute difference between prices calculated by the closed-form formula in (32) and the Monte Carlo prices, i.e. the difference between the curves in the left graph. The dashed blue line shows the difference between the closed-form formula prices and a Monte Carlo simulated price using (27) and (29) but without "freezing" in (28).

Applying Kirk's idea gives for the first futures with maturity $T_{i}$

$$
\begin{aligned}
d\left(G\left(t, T_{i}\right)+K\right)=-( & \left.G\left(t, T_{i}\right)+K\right) \frac{G\left(0, T_{i}\right)}{G\left(0, T_{i}\right)+K} \sigma\left(t, T_{i}\right)^{\top} \bar{\eta}\left(t, T_{i}\right) d t \\
& +\left(G\left(t, T_{i}\right)+K\right) \frac{G\left(0, T_{i}\right)}{G\left(0, T_{i}\right)+K} \sigma\left(t, T_{i}\right) \cdot d W_{T_{i}}(t),
\end{aligned}
$$

which is the analogue to (29). For the second futures with maturity $T_{j}$ we employ (5) in order to get the dynamics under $d W_{T_{i}}$,

$$
d G\left(t, T_{j}\right)=G\left(t, T_{j}\right) \sigma\left(t, T_{j}\right)^{\top}\left[\Gamma_{i, j-1}(t)-\bar{\eta}\left(t, T_{j}\right)\right] d t+G\left(t, T_{j}\right) \sigma\left(t, T_{j}\right) \cdot d W_{T_{i}}(t) .
$$

Using (21) we know that

$$
\Gamma_{i, j-1}(t)=\sum_{\ell=i}^{j-1} \frac{\delta L\left(0, T_{\ell}\right)}{1+\delta L(0, \ell)} \lambda\left(t, T_{\ell}\right)=\sum_{\ell=i}^{j-1} \bar{\eta}\left(t, T_{\ell+1}\right)-\bar{\eta}\left(t, T_{\ell}\right)
$$

and the dynamics of the second futures is given by

$$
d G\left(t, T_{j}\right)=-G\left(t, T_{j}\right) \sigma\left(t, T_{j}\right)^{\top} \bar{\eta}\left(t, T_{i}\right) d t+G\left(t, T_{j}\right) \sigma\left(t, T_{j}\right) \cdot d W_{T_{i}}(t),
$$


which is the analogue to (27). Solving (34) yields the relation

$$
\begin{aligned}
& \frac{G\left(t, T_{i}\right)+K}{G\left(0, T_{i}\right)+K} \exp \left\{\frac{G\left(0, T_{i}\right)}{G\left(0, T_{i}\right)+K} \int_{0}^{t} \sigma\left(s, T_{i}\right)^{\top} \bar{\eta}\left(s, T_{i}\right) d s\right\} \\
& \quad=\exp \left\{-\frac{1}{2}\left(\frac{G\left(0, T_{i}\right)}{G\left(0, T_{i}\right)+K}\right)^{2} \int_{0}^{t} \sigma\left(s, T_{i}\right)^{\top} \sigma\left(s, T_{i}\right) d s+\frac{G\left(0, T_{i}\right)}{G\left(0, T_{i}\right)+K} \int_{0}^{t} \sigma\left(s, T_{i}\right) \cdot d W_{T_{i}}(s)\right\},
\end{aligned}
$$

in which the right hand side is an exponential martingale and defines a Radon-Nikodým derivative $d \hat{\mathbb{P}} / d \mathbb{P}_{T_{i}}$. This allows us to write the spread call option prices as

$$
\begin{aligned}
C_{\text {Spread }}^{\text {Fut }}\left(0, T_{i}, T_{j}, K\right)= & B\left(0, T_{i}\right) \mathbb{E}_{T_{i}}\left[\left(G\left(T_{i}, T_{j}\right)-G\left(T_{i}, T_{i}\right)-K\right)^{+} \mid \mathcal{F}_{0}\right] \\
= & B\left(0, T_{i}\right) \mathbb{E}_{T_{i}}\left[\left(G\left(T_{i}, T_{i}\right)+K\right)\left(\frac{G\left(T_{i}, T_{j}\right)}{G\left(T_{i}, T_{i}\right)+K}-1\right)^{+} \mid \mathcal{F}_{0}\right] \\
= & B\left(0, T_{i}\right)\left(G\left(0, T_{i}\right)+K\right) \\
& \times \exp \left\{-\frac{G\left(0, T_{i}\right)}{G\left(0, T_{i}\right)+K} \int_{0}^{T_{i}} \sigma\left(s, T_{i}\right)^{\top} \bar{\eta}\left(s, T_{i}\right) d s\right\} \mathbb{E}_{\hat{\mathbb{P}}}\left[\left(Y\left(T_{i}\right)-1\right)^{+} \mid \mathcal{F}_{0}\right],
\end{aligned}
$$

now with $Y(t)=G\left(t, T_{j}\right) /\left(G\left(t, T_{i}\right)+K\right)$. The same standard techniques as for spread options on forwards yield

$$
\begin{aligned}
& C_{\text {Spread }}^{\text {Fut }}\left(0, T_{i}, T_{j}, K\right) \\
& =B\left(0, T_{i}\right)\left[G\left(0, T_{j}\right) \exp \left\{\int_{0}^{T_{i}} \sigma\left(s, T_{j}\right)^{\top} \bar{\eta}\left(s, T_{i}\right) d s\right\} N\left(d_{+}\right)\right. \\
& \left.\quad-\left(G\left(0, T_{i}\right)+K\right) \exp \left\{-\frac{G\left(0, T_{i}\right)}{G\left(0, T_{i}\right)+K} \int_{0}^{T_{i}} \sigma\left(s, T_{i}\right)^{\top} \bar{\eta}\left(s, T_{i}\right) d s\right\} N\left(d_{-}\right)\right],
\end{aligned}
$$

with

$$
\begin{aligned}
d_{ \pm} & =\frac{1}{\zeta}\left[\ln \left(\frac{G\left(0, T_{j}\right)}{G\left(0, T_{i}\right)+K}\right)+\int_{0}^{T_{i}}\left(\sigma\left(s, T_{j}\right)^{\top} \bar{\eta}\left(s, T_{i}\right)+\frac{G\left(0, T_{i}\right)}{G\left(0, T_{i}\right)+K} \sigma\left(s, T_{i}\right)^{\top} \bar{\eta}\left(s, T_{i}\right)\right) d s \pm \frac{1}{2} \zeta^{2}\right], \\
\zeta^{2} & =\int_{0}^{T_{i}}\left\|\sigma\left(s, T_{j}\right)-\frac{G\left(0, T_{i}\right)}{G\left(0, T_{i}\right)+K} \sigma\left(s, T_{i}\right)\right\|^{2} d s .
\end{aligned}
$$

\section{Conclusion}

In the present paper, a joint model of commodity and interest rate dynamics based on the LIBOR Market Model approach was constructed. We demonstrated how it can be effectively calibrated to market data, including at-the-money implied volatilities, and how less liquid instruments such as commodity spread options can be priced relative to the market using the model. In closing, one should note that although neither seasonal cycles in commodity prices nor mean reversion of the commodity price process were explicitly considered in the model construction, both of these features (well documented in the empirical literature) can be captured by the 
model, to the extent that they are reflected in current market prices: Firstly, seasonal cycles in commodity prices are anticipated by the market and thus subsumed in the term structure of futures (or forward) prices, to which the model is calibrated by construction. Secondly, mean reversion is reflected in the term structure of volatility, to which the model is also calibrated, e.g. in the presence of mean reversion of the commodity spot price process, volatility of the forward (or futures) prices increases as time to maturity decreases. Thus the martingale approach to the model construction does not preclude mean reversion simply because the objects considered explicitly are driftless - in fact, the corresponding processes under the appropriate probability measures are necessarily just as driftless in any other arbitrage-free model, including those which make mean reversion explicit in an Ornstein/Uhlenbeck process.

The main advantage of the LMM approach lies in its calibration to the market, and the model presented here opens up interesting avenues of further research (beyond the scope of the present paper) in terms of calibrating the model not just in the maturity dimension, but also in the strike dimension, along the lines of the "smile"-fitting extensions of the basic LMM discussed in Brace [2].

\section{A Volatility Integrals}

This appendix describes the calculation of integrals as in (11) and (22) for piecewise constant volatility matrices. We will focus on (22), since the integral in (11) can be obtained in the same way by setting $\bar{\eta}=\sigma$. The proposed method follows the one used in Pedersen's LMM calibration [16] to compute cap and swaption total variances.

In Section 3 a volatility matrix $U_{C} \in \mathbb{R}^{n_{f} \times d_{C}}$ has been calculated for each calendar time $t_{1}, \ldots, t_{n_{c}}$, and from LMM calibration an analogous matrix $U_{I} \in \mathbb{R}^{m_{f} \times d_{I}}$ is available for each calendar time $s_{1}, \ldots, s_{m_{c}}$. Merging over calendar times yields in each case 3-dimensional arrays $\Lambda_{C}=\left(\lambda_{i, j, k}^{C}\right) \in \mathbb{R}^{n_{c} \times n_{f} \times d}$ and $\Lambda_{I}=\left(\lambda_{i, j, k}^{I}\right) \in \mathbb{R}^{m_{c} \times m_{f} \times d_{I}}$, respectively. Forward times are given by $x_{1}, \ldots, x_{n_{f}}$ for commodity forwards and by $y_{1}, \ldots, y_{m_{f}}$ for interest forward rates. The calendar times $t_{0}=s_{0}=0$ and forward times $x_{0}=y_{0}=0$ are added for notational convenience. From these matrices of piecewise constant volatilities the integral in equation (22) has to be calculated.

The first step illustrates the computation of the $\bar{\eta}$ 's and can be omitted when calculating the integral in (11). The matrix $\Lambda_{I}$ determines the forward interest rate volatilities $\lambda(s, s+y)$ as occurring in equation (6). From these a corresponding matrix $\Theta_{I} \in \mathbb{R}^{m_{c} \times m_{f} \times d_{I}}$ with entries $\bar{\eta}_{i, j, k}$ is calculated by using relation (18). Unfortunately, the forward rates $L(t, T)$ in (18) are not known for $t>s_{0}$, but as best guess we employ the actual forward rate curve in the sense that for all future times $s_{i}>s_{0}$ an approximated forward rate curve $\bar{L}\left(s_{i}, T\right)$ is given by $L\left(s_{0}, T\right)$, essentially again making use of the standard "frozen coefficient" approximation.

In the second step, the integral for a given time to maturity $T$ in the upper integration limit is computed. We equalize the calendar time discretisation of the volatility matrices for commodity 
forwards and forward interest rates by taking the union of $t_{0}, \ldots, t_{n_{c}}$ and $s_{0}, \ldots, s_{m_{c}}$. The new calendar times will be denoted by $t_{0}=0, t_{1}, \ldots, t_{p_{c}}$. The same is done for the discretisation of times to maturity which results in a discretisation $x_{0}=0, x_{1}, \ldots, x_{p_{f}}$. The arrays $\Lambda_{C}$ and $\Theta_{I}$ are extended accordingly (i.e. by simply inserting lines for different calendar times and columns for different times to maturity, respectively, but otherwise identical entries $\lambda_{i, j, k}$ and $\bar{\eta}_{i, j, k}$ ), such that $\Lambda_{C}, \Theta_{I} \in \mathbb{R}^{p_{c} \times p_{f} \times d}$. The purpose of this extension is to have a twofold sum in formula (35) instead of a fourfold sum.

Any tuple in $\mathcal{I}=\left\{(i, j): 1 \leq i \leq p_{c}, 1 \leq j \leq p_{f}\right\}$ relates to a pair of $\sigma\left(t_{i}, t_{i}+x_{j}\right)$ and $\bar{\eta}\left(t_{i}, t_{i}+x_{j}\right)$, and in order to be relevant for the considered integral, the following inequalities need to be satisfied for some $0 \leq t \leq T$,

$$
t_{i-1} \leq t<t_{i}, x_{j-1} \leq T-t<x_{j}
$$

Equivalently, this can be written as

$$
\kappa_{i, j}:=\min \left\{t_{i}, T-x_{j-1}, T\right\}-\max \left\{t_{i-1}, T-x_{j}\right\}>0 .
$$

Hence, the integral is given by

$$
\int_{t}^{T} \sigma(u, T) \bar{\eta}(u, T) d u=\sum_{(i, j) \in \mathcal{J}} \kappa_{i, j} \sum_{k=1}^{d} \lambda_{i, j, k}^{C} \bar{\eta}_{i, j, k},
$$

where $\mathcal{J}=\left\{(i, j) \in \mathcal{I}: \kappa_{i, j}>0\right\}$.

\section{References}

[1] Black, F. (1976). The Pricing of Commodity Contracts. Journal of Financial Econometrics, Vol. 3, 167-179.

[2] Brace, A. (2007). Engineering BGM. Chapman \& Hall/CRC Financial Mathematics Series.

[3] Brace, A., Gatarek, D. and Musiela, M. (1997). The Market Model of Interest Rate Dynamics. Mathematical Finance, Vol. 7, 127-154.

[4] Choy, B., Dun, T. and Schlögl, E. (2004). Correlating Market Models. Risk, Vol. 17, No. 9, September 2004, 124-129.

[5] Cortazar, G. And Schwartz, E. S. (1994). The Valuation of Commodity Contingent Claims. Journal of Derivatives, Vol. 1, 27-39.

[6] Cox, J.C., Ingersoll, J.E. and Ross, S.A. (1981). The Relation between Forward Prices and Futures Prices. Journal of Financial Econometrics, Vol. 9, 321-346. 
[7] Gibson, R. and Schwartz, E. S. (1990). Stochastic Convenience Yield and the Pricing of Oil Contingent Claims. Journal of Finance, Vol. 45, 959-976.

[8] Heath, D., Jarrow, R. and A. Morton (1992). ond Pricing and the Term Structure of Interest Rates: A New Methodology for Contingent Claims Valuation. Econometrica, Vol. $60,77-105$.

[9] Jamshidian, F. (1997). LIBOR and Swap Market Models and Measures. Finance and Stochastics, Vol. 1, 293-330.

[10] KIRK, E. (1995). Correlation in the Energy Markets. In R. Jameson (Ed.), Managing Energy Price Risk, Risk Publications and Enron, London.

[11] Margrabe, W. (1978). The Value of an Option to Exchange One Asset For Another. Journal of Finance, Vol. 33, 177-186.

[12] Miltersen, K. (2003). Commodity Price Modelling that Matches Current Observables: A New Approach. Quantitative Finance, Vol. 3, 51-58.

[13] Miltersen, K., Sandmann, K. and Sondermann, D. (1997). Closed Form Solutions for Term Structure Derivatives with Log-Normal Interest Rates. Journal of Finance, Vol. $52,409-430$.

[14] Miltersen, K. and Schwartz, E. (1998). Pricing of Options on Commodity Futures with Stochastic Term Structures of Convenience Yield and Interest Rates. Journal of Financial and Quantitative Analysis, Vol. 33, 33-59.

[15] Musiela, M. and Rutkowski, M. (1997). Continuous-time Term Structure Models: Forward Measure Approach. Finance and Stochastics, Vol. 1, 261-291.

[16] Pedersen, M.B. (1998). Calibrating Libor Market Models. SimCorp Financial Research Working Paper.

[17] Pelsser, A. (2000). Efficient Methods for Valuing Interest Rate Derivatives. Springer.

[18] SChLÖGL, E. (2002). Arbitrage-Free Interpolation in Models of Market Observable Interest Rates. In K. Sandmann and P. Schönbucher (Eds.), Advances in Finance and Stochastics, Springer, Heidelberg.

[19] SchlöGL, E. (2002). A Multicurrency Extension of the Lognormal Interest Rate Market Models. Finance and Stochastics, Vol. 6, 173-196.

[20] Schwartz, E. S. (1982). The Pricing of Commodity-Linked Bonds. Journal of Finance, Vol. 37, 525-539. 
[21] Schwartz, E. S. (1997). The Stochastic Behavior of Commodity Prices: Implications for Valuation and Hedging. Journal of Finance, Vol. 52, 923-973. 